quatrième série-tome 41 fascicule 2 mars-avril 2008

$$
\begin{aligned}
& \text { ANNALES } \\
& \text { SCIENTIFIQUES } \\
& \text { de } \\
& \text { L'ECOLE } \\
& \text { NORMALE } \\
& \text { SUPÉRIEURE }
\end{aligned}
$$

Tien-Cuong DINH \& Nessim SIBONY

Equidistribution towards the Green current

for bolomorphic maps 
Ann. Scient. Éc. Norm. Sup.

$4^{\text {e }}$ série, t. 41, 2008, p. 307 à 336

\title{
EQUIDISTRIBUTION TOWARDS THE GREEN CURRENT FOR HOLOMORPHIC MAPS
}

\author{
BY Tien-Cuong DINH and Nessim SIBONY
}

\begin{abstract}
Let $f$ be a non-invertible holomorphic endomorphism of a projective space and $f^{n}$ its iterate of order $n$. We prove that the pull-back by $f^{n}$ of a generic (in the Zariski sense) hypersurface, properly normalized, converges to the Green current associated to $f$ when $n$ tends to infinity. We also give an analogous result for the pull-back of positive closed $(1,1)$-currents and a similar result for regular polynomial automorphisms of $\mathbb{C}^{k}$.
\end{abstract}

RÉSUMÉ. - Soient $f$ un endomorphisme holomorphe non-inversible d'un espace projectif et $f^{n}$ son itéré d'ordre $n$. Nous prouvons que l'image réciproque par $f^{n}$ d'une hypersurface générique (au sens de Zariski), proprement normalisée, converge vers le courant de Green associé à $f$ quand $n$ tend vers l'infini. Nous donnons également un résultat analogue pour les images réciproques des $(1,1)$-courants positifs fermés et un résultat similaire pour les automorphismes polynomiaux réguliers de $\mathbb{C}^{k}$.

\section{Introduction}

Let $f$ be a holomorphic endomorphism of algebraic degree $d \geq 2$ on the projective space $\mathbb{P}^{k}$. Let $\omega$ denote the Fubini-Study form on $\mathbb{P}^{k}$ normalized so that $\omega$ is cohomologous to a hyperplane or equivalently $\int_{\mathbb{P} k} \omega^{k}=1$. It is well-known that the sequence of smooth positive closed $(1,1)$-forms $d^{-n}\left(f^{n}\right)^{*}(\omega)$ converges weakly to a positive closed $(1,1)$-current $T$ of mass 1 . Moreover, $T$ has locally continuous potentials and is totally invariant, i.e. $f^{*}(T)=$ $d T$. We call $T$ the Green current of $f$. The complement of the support of $T$ is the Fatou set, i.e. the sequence $\left(f^{n}\right)$ is locally equicontinuous there. We refer the reader to the survey [29] for background. Our main results in this paper are the following theorems, where [.] denotes the current of integration on a complex variety.

TheOREm 1.1. - Let $f$ be a holomorphic endomorphism of algebraic degree $d \geq 2$ of $\mathbb{P}^{k}$ and $T$ the Green current associated to $f$. There is a proper analytic subset $\mathscr{E}$ of $\mathbb{P}^{k}$ such that if $H$ is a hypersurface of degree $s$ in $\mathbb{P}^{k}$ which does not contain any irreducible component of $\mathscr{E}$ 
then $d^{-n}\left(f^{n}\right)^{*}[H]$ converge to $s T$ in the sense of currents when $n$ tends to infinity. Moreover, $\mathscr{E}$ is totally invariant, i.e. $f^{-1}(\mathscr{E})=f(\mathscr{E})=\mathscr{E}$.

The exceptional set $\mathscr{E}$ will be explicitly constructed in Sections 6 and 7 . It is the union of totally invariant proper analytic subsets of $\mathbb{P}^{k}$ which are minimal. That is, they have no proper analytic subsets which are totally invariant, see Example 7.5. That example shows that $\mathscr{E}$ is not the maximal totally invariant analytic set. The previous result is in fact a consequence of the following one, see also Theorem 7.1 for a uniform convergence result.

Theorem 1.2. - Let $f$ be a holomorphic endomorphism of algebraic degree $d \geq 2$ of $\mathbb{P}^{k}$ and $T$ the Green current associated to $f$. There is a proper analytic subset $\mathscr{E}$ of $\mathbb{P}^{k}$, totally invariant, such that if $S$ is a positive closed $(1,1)$-current of mass 1 in $\mathbb{P}^{k}$ whose local potentials are not identically $-\infty$ on any irreducible component of $\mathscr{E}$ then $d^{-n}\left(f^{n}\right)^{*}(S) \rightarrow T$ as $n \rightarrow \infty$.

The space $\mathcal{H}_{d}$ of holomorphic maps $f$ of a given algebraic degree $d \geq 2$ is an irreducible quasi-projective manifold. We will also deduce from our study the following result due to Fornæss and the second author [18], see also [16, 29].

TheOREM 1.3. - There is a dense Zariski open subset $\mathcal{H}_{d}^{*}$ of $\mathcal{H}_{d}$ such that if $f$ is a map in $\mathcal{H}_{d}^{*}$ then $d^{-n}\left(f^{n}\right)^{*}\left(S_{n}\right) \rightarrow T$ for every sequence $\left(S_{n}\right)$ of positive closed $(1,1)$-currents of mass 1 in $\mathbb{P}^{k}$.

The rough idea in order to prove our main results is as follows. Write $S=d d^{c} u+T$. Then, the invariance of $T$ implies that $d^{-n}\left(f^{n}\right)^{*}(S)=d^{-n} d d^{c}\left(u \circ f^{n}\right)+T$. We have to show, in different situations, that $d^{-n} u \circ f^{n}$ converge to 0 in $L^{1}$. This implies that $d^{-n}\left(f^{n}\right)^{*}(S) \rightarrow T$. So, we have to study the asymptotic contraction (à la Lojasiewicz) by $f^{n}$. The main estimate is obtained using geometric estimates and convergence results for plurisubharmonic functions, see Theorem 5.1. If $d^{-n} u \circ f^{n}$ do not converge to 0 , then using that the possible contraction is limited, we construct a limit $v$ with strictly positive Lelong numbers. We then construct other functions $w_{-n}$ such that the current $d d^{c} w_{-n}+T$ has Lelong numbers $\geq \alpha_{0}>0$ and $w_{0}=d^{-n} w_{-n} \circ f^{n}$. It follows from the last identity that $w_{0}$ has positive Lelong numbers on an infinite union of analytic sets of a suitable dimension. The volume growth of these sets implies that the current associated to $w_{0}$ has too large self-intersection. This contradicts bounds due to Demailly and Méo [5, 26]. (One should notice that the Demailly-Méo estimates depend on the $L^{2}$ estimates for the $\bar{\partial}$-equation; they were recently extended to the case of compact Kähler manifolds by Vigny [33].) The previous argument has to be applied inductively on totally invariant sets for $f$, which are a priori singular and on which we inductively show the convergence to 0 , starting with sets of dimension 0 . So, we also have to develop the basics of the theory of weakly plurisubharmonic functions on singular analytic sets which is probably of independent interest. The advantage of this class of functions is that it has good compactness properties.

One may conjecture that totally invariant analytic sets should be unions of linear subspaces of $\mathbb{P}^{k}$. The case of dimension $k=2$ is proved in [3, 28]. These authors complete the result in [17]. If this were true for $k \geq 3$, our proof would be technically simpler. It is anyway interesting to carry the analysis without any assumption on the totally invariant sets since our approach may be extended to the case of meromorphic maps on compact Kähler 
manifolds. At the end of the paper, we will consider the case of regular polynomial automorphisms of $\mathbb{C}^{k}$.

The problem of convergence was first considered by Brolin for polynomials in dimension 1 and then by Lyubich, Freire, Lopes and Mañé for rational maps in $\mathbb{P}^{1}[2,19,24]$. In dimension $k=2$, Fornæss and the second author proved that $\mathscr{E}$ is empty when the local multiplicity of $f$ at every point is $\leq d-1$, see [18]. This implies Theorem 1.3 in dimension 2 for $S_{n}=S$. The proof in [18] can be extended to the general case, see also [29].

The family of hyperplanes in $\mathbb{P}^{k}$ is parametrized by a projective space of dimension $k$. It follows from Theorem 1.1 that for a hyperplane $H$, generic in the Zariski sense, we have $d^{-n}\left(f^{n}\right)^{*}[H] \rightarrow T$. Russakovskii and Shiffman have proved this result for $H$ out of a pluripolar set in the space of parameters [27]. Analogous results for subvarieties in arbitrary Kähler manifolds were proved by the authors in [10]. Concerning Theorems 1.1 and 1.2, our conditions are not optimal. Indeed, it might happen that the potentials of $S$ are identically $-\infty$ on some components of $\mathscr{E}$ and still $d^{-n}\left(f^{n}\right)^{*}(S) \rightarrow T$.

In the case of dimension $k=2$, our results (except several uniform convergences, e.g. Theorem 7.1) can be deduced from results by Favre and Jonsson. These authors say that their condition is necessary and sufficient in order to have the previous convergence, see [13], and they give needed tools for the proof in [14, p.310]. In which case, if the Lelong number of $S$ vanishes at generic points on each irreducible component of an exceptional set then $d^{-n}\left(f^{n}\right)^{*}(S) \rightarrow T$. The problem is still open in higher dimension. When the Lelong number of $S$ is 0 at every point of $\mathbb{P}^{k}$, the convergence $d^{-n}\left(f^{n}\right)^{*}(S) \rightarrow T$ was obtained by Guedj [20], see also Corollary 5.9 below. In these works, the problem of convergence is reduced to the study of sizes of images of balls under iterates of $f$. This approach was first used in [16, 18].

Recall that the self-intersection $T^{p}:=T \wedge \cdots \wedge T, p$ times, defines a positive closed $(p, p)$ current which is totally invariant, i.e. $f^{*}\left(T^{p}\right)=d^{p} T^{p}$, see [11] for the pull-back operator on currents. It is natural to consider the analogous equidistribution problem towards $T^{p}$.

CONJECTURe 1.4. - Let $f$ be a holomorphic endomorphism of $\mathbb{P}^{k}$ of algebraic degree $d \geq 2$ and $T$ its Green current. Then $d^{-p n}\left(f^{n}\right)^{*}[H]$ converge to $s T^{p}$ for every analytic subset $H$ of $\mathbb{P}^{k}$ of pure codimension $p$ and of degree $s$ which is generic. Here, $H$ is generic if either $H \cap E=\varnothing$ or codim $H \cap E=p+\operatorname{codim} E$ for any irreducible component $E$ of every totally invariant analytic subset of $\mathbb{P}^{k}$.

We will see later that there are only finitely many analytic sets which are totally invariant. Theorem 1.1 proves the conjecture for $p=1$. Indeed, in that case, it is equivalent to check the condition for minimal totally invariant analytic sets. For $p=k$, the measure $\mu:=T^{k}$ is the unique invariant measure of maximal entropy, see [18, 1, 29]. In this case, the conjecture was proved by the authors in [9]. Weaker results in this direction were obtained in [18] and [1]. We will give some details in Theorem 6.6. For $2 \leq p \leq k-1$, the authors have proved in [12] that for $f$ in a Zariski dense open set $\mathcal{H}_{d}^{\prime} \subset \mathcal{H}_{d}$, there is no proper analytic subset of $\mathbb{P}^{k}$ which is totally invariant and that the conjecture holds. Indeed, a version of Theorem 1.3 is proved. 


\section{Plurisubharmonic functions}

We refer the reader to [22, 6, 10] for the basic properties of plurisubharmonic (psh for short) and quasi-psh functions on smooth manifolds. In order to study the Levi problem for analytic spaces $X$, the psh functions which are considered, are the restrictions of psh functions on an open set of $\mathbb{C}^{k}$ for a local embedding of $X$. Let $u: X \rightarrow \mathbb{R} \cup\{-\infty\}$ be an upper semi-continuous function which is not identically equal to $-\infty$ on any irreducible component of $X$. Fornæss-Narasimhan proved that if $u$ is subharmonic or equal to $-\infty$ on any holomorphic disc in $X$, then $u$ is psh in the above sense [15]. However, this class does not satisfy good compactness properties which are useful in our analysis. Assume that $X$ is an analytic space of pure dimension $p$. Let $\operatorname{reg}(X)$ and $\operatorname{sing}(X)$ denote the regular and the singular parts of $X$. We consider the following weaker notion of psh functions which is modeled after the notion of weakly holomorphic functions. The class has good compactness properties.

Definition 2.1. - A function $v: X \rightarrow \mathbb{R} \cup\{-\infty\}$ is wpsh if

(a) $v$ is psh on $X \backslash \operatorname{sing}(X)$;

(b) for $a \in \operatorname{sing}(X), v(a)=\lim \sup v(x)$ with $x \in \operatorname{reg}(X)$ and $x \rightarrow a$.

Fornæss-Narasimhan's theorem implies that psh functions are wpsh. Wpsh functions are psh when $X$ is smooth. One should notice that the restriction of a wpsh function to an irreducible component of $X$ is not necessarily wpsh. For example, consider $X=\{x y=0\}$ in the unit ball of $\mathbb{C}^{2}$, let $v=0$ on $\{x=0\} \backslash(0,0)$ and $v=1$ on $\{y=0\}$, then $v$ is wpsh on $X$ but its restriction to $\{x=0\}$ is not wpsh. Consider the (strongly) psh function $v_{n}:=|x|^{1 / n}$ on $X$. The sequence $v_{n}$ converges to $v$ in $L^{1}(X)$. So, psh functions on analytic sets do not have good compactness properties.

Proposition 2.2. - Let $Z \subset X$ be an analytic subset of dimension $\leq p-1$ and $v^{\prime} a$ wpsh function on $X \backslash Z$. If $v^{\prime}$ is locally bounded from above near $Z$ then there is a unique wpsh function $v$ on $X$ equal to $v^{\prime}$ outside $Z$.

Proof. - The extension to a psh function on $\operatorname{reg}(X)$ is well-known. So, we can assume that $Z \subset \operatorname{sing}(X)$. Condition (b) in Definition 2.1 implies the uniqueness of the extension of $v^{\prime}$. Define $v(a):=\lim \sup v(x)$ with $x \notin Z$ and $x \rightarrow a$. It is clear that $v=v^{\prime}$ out of $Z$ and $v$ satisfies the conditions in Definition 2.1.

Now assume for simplicity that $X$ is an analytic subset of pure dimension $p$ of an open set $U$ in $\mathbb{C}^{k}$. The general case can be deduced from this one. The following results give characterizations of wpsh functions.

Proposition 2.3. - Let $\pi: \widetilde{X} \rightarrow X \subset U$ be a desingularization of $X$. If $v$ is a wpsh function on $X$ then there is a psh function $\widetilde{v}$ on $\widetilde{X}$ such that $v(x)=\max _{\pi^{-1}(x)} \widetilde{v}$ for $x \in X$. Conversely, if $\widetilde{v}$ is psh on $\widetilde{X}$ then $x \mapsto \max _{\pi^{-1}(x)} \widetilde{v}$ defines a wpsh function on $X$. 
Proof. - Define $\widetilde{v}:=v \circ \pi$ outside the analytic set $\pi^{-1}(\operatorname{sing}(X))$. This function is psh and is locally bounded above near $\pi^{-1}(\operatorname{sing}(X))$. We can extend it to a psh function on $\widetilde{X}$ that we also denote by $\widetilde{v}$. For $x \in X, \pi^{-1}(x)$ is compact. The maximum principle implies that $\widetilde{v}$ is constant on each irreducible component of $\pi^{-1}(x)$. From the definition of wpsh function, we get $v(x)=\max _{\pi^{-1}(x)} \widetilde{v}$. The second assertion in the proposition follows from the definition of wpsh functions.

A theorem of Lelong says that the integration on $\operatorname{reg}(X)$ defines a positive closed $(k-p, k-p)$-current $[X]$ on $U$, see $[23,6]$. Let $z$ denote the coordinates in $\mathbb{C}^{k}$.

Proposition 2.4. - A function $v: X \rightarrow \mathbb{R} \cup\{-\infty\}$ is wpsh if and only if the following properties are satisfied:

(a) $v$ is in $L_{l o c}^{1}(X)$, i.e. $\int_{K}|v|\left(d d^{c}|z|^{2}\right)^{p}<+\infty$ for any compact set $K \subset X$.

(b) $v$ is strongly upper semi-continuous, i.e. for any $a \in X$ and any full measure subset $X^{\prime} \subset X$ we have $v(a)=\lim \sup v(x)$ with $x \in X^{\prime}$ and $x \rightarrow a$.

(c) $d d^{c}(v[X])$ is a positive current on $U$.

Proof. - We use the notations in Proposition 2.3. The proposition is known for smooth manifolds, see [6]. Assume that $v$ is wpsh. The function $\widetilde{v}$ defined above satisfies properties (a), (b) and (c) on $\widetilde{X}$. It follows that $v$ satisfies (a) and (b) on $X$. Since $d d^{c}(v[X])=$ $\pi_{*}\left(d d^{c}(\widetilde{v}[\widetilde{X}])\right), d d^{c}(v[X])$ is positive. Hence, $v$ satisfies (c).

Conversely, Properties (a)-(c) imply that $v$ is psh on $\operatorname{reg}(X)$. Then, Property (b) implies that $v$ satisfies the conditions of Definition 2.1.

Proposition 2.5. - Let $\left(v_{n}\right)$ be a sequence of wpsh functions on $X$, locally uniformly bounded from above. Then, there is a subsequence $\left(v_{n_{i}}\right)$ satisfying one of the following properties:

(a) There is an irreducible component $Y$ of $X$ such that $\left(v_{n_{i}}\right)$ converges uniformly to $-\infty$ on $K \backslash \operatorname{sing}(X)$ for any compact set $K \subset Y$.

(b) $\left(v_{n_{i}}\right)$ converges in $L_{l o c}^{q}(X)$ to a wpsh function $v$ for every $1 \leq q<+\infty$.

In the last case, $\lim \sup v_{n_{i}} \leq v$ on $X$ with equality almost everywhere.

Proof. - Let $\pi: \widetilde{X} \rightarrow X \subset U$ be as above. We extend the functions $v_{n} \circ \pi$, which are psh on $\pi^{-1}(\operatorname{reg}(X))$ to psh functions $\widetilde{v}_{n}$ on $\widetilde{X}$. Recall that $v_{n}(x)=\max _{\pi^{-1}(x)} \widetilde{v}_{n}$. Now, since the proposition holds for smooth manifolds, it is enough to apply it to $\left(\widetilde{v}_{n}\right)$. If a psh function $\widetilde{v}$ is a limit value of $\left(\widetilde{v}_{n}\right)$ in $L_{l o c}^{q}(\widetilde{X})$, the function $v$, defined by $v(x):=\max _{\pi^{-1}(x)} \widetilde{v}$, satisfies the property (b) in the proposition. If not, $\widetilde{v}_{n}$ converges to $-\infty$ locally uniformly on some component of $\widetilde{X}$ and the property (a) holds.

The following result is the classical Hartogs' lemma when $X$ is smooth [22].

Lemma 2.6. - Let $\left(v_{n}\right)$ be a sequence of wpsh functions on $X$. Let u be a continuous function on $X$ such that $\lim \sup v_{n}<u$. Then for every compact set $K \subset X, v_{n}<u$ on $K$ for $n$ large enough. This holds in particular, if $\left(v_{n}\right)$ converges to a wpsh function $v$ in $L_{l o c}^{1}(X)$ and $v<u$. 
Proof. - Let $\pi$ and $\widetilde{v}_{n}$ be defined as above. These functions $\widetilde{v}_{n}$ are psh on $\widetilde{X}$. Define $\widetilde{u}:=u \circ \pi$. It is clear that $\widetilde{u}$ is continuous and that $\lim \sup \widetilde{v}_{n} \leq \lim \sup v_{n} \circ \pi<\widetilde{u}$. We only have to apply the classical Hartogs' lemma in order to obtain $\widetilde{v}_{n}<\widetilde{u}$ on $\pi^{-1}(K)$ for $n$ large enough. This implies the result. The last assertion in the lemma is a consequence of Proposition 2.5.

The following lemma will be useful.

Lemma 2.7. - Let $\mathscr{G}$ be a family of psh functions on $U$ locally uniformly bounded from above. Assume that for each irreducible component of $X$ there is an analytic subset $Z$ such that the restriction of $\mathscr{G}$ to $Z$ is bounded in $L_{l o c}^{1}(Z)$. Then, the restriction of $\mathscr{G}$ to $X$ is bounded in $L_{l o c}^{1}(X)$.

Proof. - We can assume that $X$ is irreducible. For $\left(v_{n}\right) \subset \mathscr{G}$, define the psh functions $\widetilde{v}_{n}$ on $\widetilde{X}$ as above. It is clear that $\widetilde{v}_{n}$ are locally uniformly bounded from above. Let $W \Subset U$ be an open set which intersects $Z$. The maximal value of $\widetilde{v}_{n}$ on $\pi^{-1}(Z \cap \bar{W})$ is equal to the maximal value of $v_{n}$ on $Z \cap \bar{W}$. It follows from the hypothesis that no subsequence of $\left(\widetilde{v}_{n}\right)$ converges uniformly on compact sets to $-\infty$. Proposition 2.5 applied to $\left(\widetilde{v}_{n}\right)$, implies that this sequence is bounded in $L_{l o c}^{1}(\widetilde{X})$. Applying again Proposition 2.5 to $\left(v_{n}\right)$ gives the lemma.

Let $R$ be a positive closed $(1,1)$-current on $U$ with continuous local potentials, i.e. locally $R=d d^{c} v$ with $v$ psh and continuous. Let $R^{\prime}$ be a positive closed $(k-p, k-p)$-current on $U, 1 \leq p \leq k-1$. Recall that we can define their intersection by $R \wedge R^{\prime}:=d d^{c}\left(v R^{\prime}\right)$ where $v$ is a local potential of $R$ as above. This is a positive closed $(k-p+1, k-p+1)$-current on $U$ which depends continuously on $R^{\prime}$. The definition is independent of the choice of $v$. By induction, if $R_{1}, \ldots, R_{p}$ are positive closed $(1,1)$-currents with continuous local potentials, the intersection $\nu:=R_{1} \wedge \cdots \wedge R_{p} \wedge[X]$ is a positive measure with support in $X$. This product is symmetric with respect to $R_{1}, \ldots, R_{p}$.

Proposition 2.8. - For every compact sets $K$ and $K^{\prime}$ with $K \Subset K^{\prime} \subset X$, there is a constant $c>0$ such that if $u$ is wpsh on $X$ we have

$$
\max _{K} u \leq c\|u\|_{L^{1}\left(K^{\prime}\right)} \quad \text { and } \quad \int_{K}|u| d \nu \leq c\|u\|_{L^{1}\left(K^{\prime}\right)} .
$$

In particular, $\nu$ has no mass on analytic subsets of dimension $\leq p-1$ of $X$.

Proof. - Choose a compact set $L$ such that $K \Subset L \Subset K^{\prime}$ and a neighbourhood $W$ of $\operatorname{sing}(X)$ small enough. If $a$ is a point in $K \cap W$, then we can find a Riemann surface in $X$ containing $a$ and having boundary in $L \backslash W$. Indeed, it is enough to consider the intersection of $X$ with a suitable linear plane $P$ of dimension $k-p+1$ passing through $a$. The maximum principle applied to the lift of $u$ to $\widetilde{X}$ (defined above) implies that $u(a) \leq \max _{L \backslash W} u$ and hence $\max _{K} u \leq \max _{L \backslash W} u$. Since $L \backslash W \subset \operatorname{reg}(X)$, the submean inequality for psh functions on smooth manifolds implies that $\max _{L \backslash W} u \leq c\|u\|_{L^{1}\left(K^{\prime}\right)}$ for some constant $c>0$. Hence, $\max _{K} u \leq c\|u\|_{L^{1}\left(K^{\prime}\right)}$.

We prove now the second inequality. Replacing $u$ by $u-c\|u\|_{L^{1}\left(K^{\prime}\right)}$ allows us to assume that $u \leq 0$ on $K$. Since the problem is local, we can assume that $R_{i}=d d^{c} v_{i}$ with $v_{i}$ continuous on $U$. Moreover, we can approximate $v_{i}$ by decreasing sequences $\left(v_{i, n}\right)$ of smooth psh 
functions. Define $R_{i, n}:=d d^{c} v_{i, n}$. It is well-known that $\nu_{n}:=R_{1, n} \wedge \cdots \wedge R_{p, n} \wedge[X]$ converge to $\nu$ in the sense of measures. Using the same arguments as in the Chern-Levine-Nirenberg inequalities $[4,6,29]$ yields

$$
\int_{K} u d \nu_{n} \geq-c^{\prime}\left\|v_{1, n}\right\|_{L^{\infty}\left(K^{\prime}\right)} \cdots\left\|v_{p, n}\right\|_{L^{\infty}\left(K^{\prime}\right)}\|u\|_{L^{1}\left(K^{\prime}\right)}
$$

where $c^{\prime}>0$ is independent of $n$. When $n \rightarrow \infty$, since $\nu_{n} \rightarrow \nu$ and since $u$ is upper semicontinuous, we obtain

$$
\int_{K} u d \nu \geq-c^{\prime}\left\|v_{1}\right\|_{L^{\infty}\left(K^{\prime}\right)} \cdots\left\|v_{p}\right\|_{L^{\infty}\left(K^{\prime}\right)}\|u\|_{L^{1}\left(K^{\prime}\right)} .
$$

This implies the second inequality in the proposition.

Let $Y$ be an analytic subset of $X$ of dimension $\leq p-1$. Then, there is a psh function $u^{\prime}$ on $U$ such that $\left\{u^{\prime}=-\infty\right\}=Y$. The last inequality, applied to the restriction of $u^{\prime}$ to $X$, implies $\nu(Y)=0$.

\section{Modulo $T$ plurisubharmonic functions}

We are going to develop in this section the analogue in the compact case of the local theory in Section 2. Consider a (compact) analytic subset $X$ of $\mathbb{P}^{k}$ of pure dimension $p$. Recall that the Green current $T$ of $f$ has locally continuous potentials. Observe that in what follows (except for Lemma 3.8, Corollary 3.9 and Remark 3.10), $T$ could be an arbitrary positive closed $(1,1)$-current of mass 1 with continuous potentials, and $\mathbb{P}^{k}$ could be replaced by any compact Kähler manifold. We will use the following notion that allows us to simplify the notations.

Definition 3.1. - A function $u: X \rightarrow \mathbb{R} \cup\{-\infty\}$ is wpsh modulo $T$ if locally it is the difference of a wpsh function on $X$ and a potential of $T$. If $X$ is smooth, we say that $u$ is $p s h$ modulo $T$.

The following result is a consequence of Proposition 2.4.

Proposition 3.2. - A function $u: X \rightarrow \mathbb{R} \cup\{-\infty\}$ is wpsh modulo $T$ if and only if the following properties are satisfied

(a) $u$ is in $L^{1}(X)$, i.e. $\int_{X}|u| \omega^{p}<+\infty$.

(b) $u$ is strongly upper semi-continuous.

(c) $d d^{c}(u[X]) \geq-T \wedge[X]$ on $\mathbb{P}^{k}$.

Note that if $u$ is a modulo $T$ wpsh function, $d d^{c}(u[X])+T \wedge[X]$ is a positive closed current of bidegree $(k-p+1, k-p+1)$ supported on $X$. If $S$ is a positive closed $(1,1)$-current on $\mathbb{P}^{k}$ of mass 1 , then it is cohomologous to $T$ and we can write $S=T+d d^{c} u$ where $u$ is a modulo $T$ psh function on $\mathbb{P}^{k}$. The restriction of such a function $u$ to $X$ is either wpsh modulo $T$ or equal to $-\infty$ on at least one irreducible component of $X$.

The following proposition is a consequence of Proposition 2.5. 
Proposition 3.3. - Let $\left(u_{n}\right)$ be a sequence of modulo $T$ wpsh functions on $X$, uniformly bounded from above. Then there is a subsequence $\left(u_{n_{i}}\right)$ satisfying one of the following properties:

(1) There is an irreducible component $Y$ of $X$ such that $\left(u_{n_{i}}\right)$ converges uniformly to $-\infty$ on $Y \backslash \operatorname{sing}(X)$.

(2) $\left(u_{n_{i}}\right)$ converges in $L^{q}(X)$ to a modulo $T$ wpsh function $u$ for every $1 \leq q<+\infty$.

In the last case, $\lim \sup u_{n_{i}} \leq u$ on $X$ with equality almost everywhere.

The Hartogs' lemma 2.6 implies the following.

Lemma 3.4. - Let $\left(u_{n}\right)$ be a sequence of modulo $T$ wpsh functions on $X$ converging in $L^{1}(X)$ to a modulo $T$ wpsh function $u$. If $w$ is a continuous function on $X$ such that $u<w$, then $u_{n}<w$ for $n$ large enough.

The following lemma is deduced from Lemma 2.7.

LeMma 3.5. - Let $\mathscr{G}$ be a family of modulo $T$ psh functions on $\mathbb{P}^{k}$ uniformly bounded from above. Assume that each irreducible component of $X$ contains an analytic subset $Y$ such that the restriction of $\mathscr{G}$ to $Y$ is bounded in $L^{1}(Y)$. Then, the restriction of $\mathscr{G}$ to $X$ is bounded in $L^{1}(X)$.

Define a positive measure supported on $X$ by $\mu_{X}:=T^{p} \wedge[X]$. By Bézout's theorem, the mass of $\mu_{X}$ is equal to the degree of $X$. The same argument implies that $\mu_{X}$ has positive mass on any irreducible component of $X$. The following result is a consequence of Proposition 2.8.

Proposition 3.6. - There is a constant $c>0$ such that if $u$ is a modulo $T$ wpsh function on $X$ then

$$
\max _{X} u \leq c\left(1+\|u\|_{L^{1}(X)}\right) \quad \text { and } \quad \int|u| d \mu_{X} \leq c\left(1+\|u\|_{L^{1}(X)}\right) .
$$

In particular, $\mu_{X}$ has no mass on analytic subsets of dimension $\leq p-1$ of $X$.

We also have the following useful Proposition and Lemma.

Proposition 3.7. - A family $\mathscr{G}$ of modulo $T$ wpsh functions on $X$ is bounded in $L^{1}(X)$ if and only if there is a constant $c>0$ such that $\left|\int u d \mu_{Y}\right| \leq c$ for $u \in \mathscr{G}$ and for any irreducible component $Y$ of $X$.

Proof. - Proposition 3.6 implies that $\mu_{Y}$ has no mass on $\operatorname{sing}(X)$. If $\mathscr{G}$ is bounded in $L^{1}(X)$ then it is bounded in $L^{1}(Y)$. We have seen that the restriction of $u \in \mathscr{G}$ to $Y$ is equal outside $\operatorname{sing}(X)$ to a modulo $T$ wpsh function on $Y$. By Proposition 3.6, there is a constant $c>0$ such that $\left|\int u d \mu_{Y}\right| \leq c$ for $u \in \mathscr{G}$.

Conversely, assume that $\left|\int u d \mu_{Y}\right| \leq c$ for $u \in \mathscr{G}$ and for any irreducible component $Y$ of $X$. Since $\mu_{Y}$ has no mass on $\operatorname{sing}(X)$, we can replace $X$ by $Y$ and assume that $X$ is irreducible. Define $m_{u}:=\max _{X} u$ and $v:=u-m_{u}$. Since $\max _{X} v=0$, Proposition 3.3 implies that the family of such functions $v$ is bounded in $L^{1}(X)$, see also Definition 2.1(b). On the other hand, we have

$$
\left|m_{u}\right|\left\|\mu_{X}\right\|=\left|\int u d \mu_{X}-\int v d \mu_{X}\right| \leq c+\left|\int v d \mu_{X}\right| .
$$

$4^{\mathrm{e}}$ SÉRIE - TOME $41-2008-\mathrm{N}^{\mathrm{o}} 2$ 
This and Proposition 3.6, applied to $v$, imply that $\left|m_{u}\right|$ is bounded. Since $u=m_{u}+v$, we obtain that $\mathscr{G}$ is bounded in $L^{1}(X)$.

Lemma 3.8. - Let $u$ be a modulo $T$ wpsh function on $X$. If $X$ is invariant by $f$, i.e. $f(X)=X$, then $d^{-1} u \circ f$ is equal out of $\operatorname{sing}(X) \cup f^{-1}(\operatorname{sing}(X))$ to a modulo $T$ wpsh function $w$ on $X$. Moreover, $w$ depends continuously on $u$.

Proof. - Consider a point $x \in X$ out of $\operatorname{sing}(X) \cup f^{-1}(\operatorname{sing}(X))$. Since $T$ is totally invariant, if $v$ is a potential of $T$ in a neighbourhood $V$ of $f(x)$ then $d^{-1} v \circ f$ is a potential of $T$ in a neighbourhood $U$ of $x$. Since the function $u+v$ is psh on $X \cap V, d^{-1}(u \circ f+v \circ f)$ is psh on $X \cap U$. Hence, $d d^{c}\left(\left(d^{-1} u \circ f\right)[X]\right) \geq-T \wedge[X]$ out of $\operatorname{sing}(X) \cup f^{-1}(\operatorname{sing}(X))$. On the other hand, since $u$ is bounded from above, $d^{-1} u \circ f$ is bounded from above. Proposition $2.2 \mathrm{im}$ plies the existence of $w$. That $w$ depends continuously on $u$ follows from Proposition 3.3.

Corollary 3.9. - Assume that $X$ is invariant. Let $\mathscr{G}$ be a family of modulo $T$ wpsh functions on $X$, bounded in $L^{1}(X)$. Then, the family of modulo $T$ wpsh functions on $X$ which are equal almost everywhere to $d^{-n} u \circ f^{n}$ with $n \geq 0$ and $u \in \mathscr{G}$, is bounded in $L^{1}(X)$. Moreover, if a modulo $T$ wpsh function $u$ on $X$ is a limit value of $\left(d^{-n} u_{n} \circ f^{n}\right)$ in $L^{1}(X)$ with $u_{n} \in \mathscr{G}$, then $u \leq 0$ on $X$ and $u=0$ on $\operatorname{supp}\left(\mu_{X}\right)$. The sequence $\left(d^{-n} u_{n} \circ f^{n}\right)$ converges to 0 in $L^{1}\left(\mu_{X}\right)$.

Proof. - Replacing $f$ by an iterate $f^{n}$ allows us to assume that $f$ fixes all the irreducible components of $X$. So, we can assume that $X$ is irreducible. For the first assertion, by Proposition 3.6, we can subtract from each $u$ a constant in order that $\max _{X} u=0$. So, we can assume that $\mathscr{G}$ is the set of such functions $u$. This is a bounded set in $L^{1}(X)$. All the functions $d^{-n} u \circ f^{n}$ are equal almost everywhere to functions in $\mathscr{G}$. The first assertion follows.

For the second assertion, by Lemma 3.8, $d^{-n} u_{n} \circ f^{n}$ is equal outside an analytic set to a modulo $T$ wpsh function $v_{n}$ on $X$. Propositions 3.3 and 3.6 imply that $u_{n} \leq A$ and $\int\left|u_{n}\right| d \mu_{X} \leq A$ for some constant $A>0$. It follows that $v_{n} \leq d^{-n} A$, see also Proposition 3.2(b), and then $\lim \sup v_{n} \leq 0$. Hence, $u \leq 0$. On the other hand, since $X$ is invariant and $T$ is totally invariant, we have $\left(f^{n}\right)_{*}\left(\mu_{X}\right)=\mu_{X}$ and

$$
\left|\int\left(d^{-n} u_{n} \circ f^{n}\right) d \mu_{X}\right|=d^{-n}\left|\int u_{n} d\left(f^{n}\right)_{*}\left(\mu_{X}\right)\right|=d^{-n}\left|\int u_{n} d \mu_{X}\right| \leq d^{-n} A .
$$

Hence, $\int v_{n} d \mu_{X} \rightarrow 0$. By Propositions 3.7 and 3.6, $\left(v_{n}\right)$ is bounded from above. This allows us to apply the last assertion in Proposition 3.3. We deduce from Fatou's lemma and the convergence $\int v_{n} d \mu_{X} \rightarrow 0$, that $\int u d \mu_{X} \geq 0$. This and the inequality $u \leq 0$ imply that $u=0 \mu_{X}$-almost everywhere. By upper semi-continuity, $u=0$ on $\operatorname{supp}\left(\mu_{X}\right)$.

REMARK 3.10. - Assume that $f$ is chaotic, i.e. the support of the Green measure $\mu$ of $f$ is equal to $\mathbb{P}^{k}$. Then, the previous corollary gives us a simple proof of the following property: for all positive closed $(1,1)$-currents $S_{n}$ of mass 1 on $\mathbb{P}^{k}$, we have $d^{-n}\left(f^{n}\right)^{*}\left(S_{n}\right) \rightarrow T$. Indeed, we can write $S_{n}=T+d d^{c} u_{n}$ with $u_{n}$ bounded in $L^{1}\left(\mathbb{P}^{k}\right)$, and hence $d^{-n} u_{n} \circ f^{n}$ converge to 0 . 


\section{Lelong numbers}

In this section, we recall some properties of the Lelong numbers of currents and of plurisubharmonic functions, see [6] for a systematic exposition.

Let $R$ be a positive closed $(p, p)$-current on an open set $U$ of $\mathbb{C}^{k}$. Let $z$ denote the coordinates in $\mathbb{C}^{k}$ and $B_{a}(r)$ the ball of center $a$ and of radius $r$. Then, $R \wedge\left(d d^{c}\|z\|^{2}\right)^{k-p}$ is a positive measure on $U$. Define for $a \in U$

$$
\nu(R, a, r):=\frac{\left\|R \wedge\left(d d^{c}\|z\|^{2}\right)^{k-p}\right\|_{B_{a}(r)}}{\pi^{k-p} r^{2(k-p)}} .
$$

When $r$ decreases to $0, \nu(R, a, r)$ is decreasing and the Lelong number of $R$ at $a$ is the limit

$$
\nu(R, a):=\lim _{r \rightarrow 0} \nu(R, a, r) .
$$

The property that $\nu(R, a, r)$ is decreasing implies the following property that we will use later: if $R_{n} \rightarrow R$ and $a_{n} \rightarrow a$, then $\lim \sup \nu\left(R_{n}, a_{n}\right) \leq \nu(R, a)$.

The Lelong number $\nu(R, a)$ is also the mass of the measure $R \wedge\left(d d^{c} \log \|z-a\|\right)^{k-p}$ at $a$. It does not depend on the coordinates. So, we can define the Lelong number for currents on any manifold. If $R$ is the current of integration on an analytic set $V$, by Thie's theorem, $\nu(R, a)$ is equal to the multiplicity of $V$ at $a$. Recall also a theorem of Siu which says that for $c>0$ the level set $\{\nu(R, a) \geq c\}$ is an analytic subset of dimension $\leq k-p$ of $U$.

Let $S$ be a current of bidegree $(1,1)$ and $v$ a potential of $S$ on $U$. Define the Lelong number of $v$ at $a$ by $\nu(v, a):=\nu(S, a)$. We also have

$$
\nu(v, a)=\lim _{r \rightarrow 0} \frac{\sup _{B_{a}(r)} v(z)}{\log r} .
$$

The function $\log r \mapsto \sup _{B_{a}(r)} v$ is increasing and convex with respect to $\log r$. It follows that if $v$ is defined on $B_{a}(1)$ and is negative, the fraction in (1) is decreasing when $r$ decreases to 0 . So, if two psh functions differ by a locally bounded function, they have the same Lelong number at every point. Moreover, identity (1) allows to define the Lelong number for every function which locally differs from a psh function by a bounded function.

Let $X$ be an analytic subset of pure dimension $p$ in $U$ and $u$ a wpsh function on $X$. Then, $S^{X}:=d d^{c}(u[X])$ is a positive closed $(k-p+1, k-p+1)$-current on $U$. Define

$$
\nu_{X}(u, a):=\nu\left(S^{X}, a\right) .
$$

When $X$ is smooth at $a$, we can also define a positive closed $(1,1)$-current on a neighbourhood of $a$ in $X$ by $S_{X}:=d d^{c} u$. We have $\nu_{X}(u, a)=\nu\left(S_{X}, a\right)$ where the last Lelong number is defined on $X$.

Consider a proper finite holomorphic map $h: U^{\prime} \rightarrow U$ between an open set $U^{\prime}$ of $\mathbb{C}^{k}$ and $U$. Let $X^{\prime}$ be an analytic subset of pure dimension $p$ of $U^{\prime}$ such that $h\left(X^{\prime}\right)=X$, and $a^{\prime} \in U^{\prime}$ a point such that $h\left(a^{\prime}\right)=a$. It follows from Proposition 2.2 that $u \circ h$ is equal almost everywhere to a wpsh function $u^{\prime}$ on $X^{\prime}$. The continuous dependence of $u^{\prime}$ with respect to $u$ is proved as in Lemma 3.8.

Proposition 4.1. - Let $\delta$ denote the local topological degree of $h$ at $a^{\prime}$. Then

$$
\delta^{-k} \nu_{X}(u, a) \leq \nu_{X^{\prime}}\left(u^{\prime}, a^{\prime}\right) \leq \delta \nu_{X}(u, a) .
$$

$4^{\mathrm{e}}$ SÉRIE - TOME $41-2008-\mathrm{N}^{\mathrm{o}} 2$ 
Proof. - Recall that $X$ and $X^{\prime}$ may be reducible and singular, but one can work on each irreducible component separately. We deduce from the identity $h\left(X^{\prime}\right)=X$ and from the definition of $\delta$ that near $a$ :

$$
d d^{c}(u[X]) \leq h_{*}\left(d d^{c}\left(u^{\prime}\left[X^{\prime}\right]\right)\right) \leq \delta d d^{c}(u[X]) .
$$

Hence,

$$
\nu\left(d d^{c}(u[X]), a\right) \leq \nu\left(h_{*}\left(d d^{c}\left(u^{\prime}\left[X^{\prime}\right]\right)\right), a\right) \leq \delta \nu\left(d d^{c}(u[X]), a\right) .
$$

On the other hand, by Theorems 9.9 and 9.12 in [6], we have

$$
\nu\left(d d^{c}\left(u^{\prime}\left[X^{\prime}\right]\right), a^{\prime}\right) \leq \nu\left(h_{*}\left(d d^{c}\left(u^{\prime}\left[X^{\prime}\right]\right)\right), a\right) \leq \delta^{k} \nu\left(d d^{c}\left(u^{\prime}\left[X^{\prime}\right]\right), a^{\prime}\right) .
$$

The inequalities in the proposition follow from (2) and (3).

Let $B_{a}^{X}(r)$ denote the connected component of $B_{a}(r) \cap X$ which contains $a$. We call it the ball of center $a$ and of radius $r$ in $X$.

Proposition 4.2. - Let $\mathscr{G}$ be a family of wpsh functions on $X$ which is compact in $L_{\text {loc }}^{1}(X)$. Let $\delta>0$ such that $\nu_{X}(u, a)<\delta$ for $u \in \mathscr{G}$ and $a \in X$. Then, for any compact set $K \subset X$, there exist constants $c>0$ and $A>0$ such that

$$
\sup _{B_{a}^{X}(r)} u \geq c \delta \log r-A \quad \text { for } u \in \mathscr{G}, a \in K \text { and } 0<r<1 .
$$

Moreover, the constant $c$ is independent of $\mathscr{G}$ and of $\delta$.

Proof. - Reducing $U$ allows to assume that $\mathscr{G}$ is bounded in $L^{1}(X)$ and $\nu_{X}(u, a) \leq \delta-\varepsilon$, $\varepsilon>0$, on $X$ for every $u \in \mathscr{G}$. Moreover, by Proposition 2.8, $\mathscr{G}$ is uniformly bounded from above. So, we can assume that $u \leq 0$ for every $u \in \mathscr{G}$. If $0<r_{0}<1$ is fixed and $r_{0}<r<1$, the fact that $\mathscr{G}$ is bounded in $L^{1}(X)$ implies that $\sup _{B_{a}^{X}(r)} u \geq-A$ for every $a \in K$ where $A>0$ is a constant. Hence, it is enough to consider $r$ small.

We first consider the case where $X$ is smooth. Since the problem is local we can assume that $X$ is a ball in $\mathbb{C}^{p}$. Up to a dilation of coordinates, we can assume that the distance between $K$ and $\partial X$ is larger than 1. Define

$$
s(u, a, r):=\frac{\sup _{B_{a}(r) \cap X} u}{\log r} .
$$

Hence, for $a \in K$ and for $0<r<1, s(u, a, r)$ decreases to $\nu(u, a)$ when $r$ decreases to 0 . For every $(a, u) \in K \times \mathscr{G}$, since $\nu(u, a) \leq \delta-\varepsilon$, there is an $r>0$ such that $s\left(u, a, r^{\prime}\right) \leq \delta-\varepsilon / 2$ for $r^{\prime} \leq 2 r$. It follows that if a psh function $v$ on $X$ is close enough to $u$ then $s(v, a, r) \leq \delta-\varepsilon / 4$, see Lemma 2.6. We then deduce from the definition of $s(v, a, r)$ that if $b$ is close enough to $a$ and if $r^{\prime \prime}:=r-|b-a|$ then

$$
s(v, b, r) \leq \frac{\log r^{\prime \prime}}{\log r} s\left(v, a, r^{\prime \prime}\right) \leq \frac{\log r^{\prime \prime}}{\log r} s(v, a, r) \leq \delta .
$$

The fact that $s(v, b, r)$ is increasing implies that $s\left(v, b, r^{\prime}\right) \leq \delta$ for $r^{\prime} \leq r$ and for $(b, v)$ in a neighbourhood of $(a, u)$. Since $K \times \mathscr{G}$ is compact, if $r$ is small enough, the inequality $s(u, a, r) \leq \delta$ holds for every $(a, u) \in K \times \mathscr{G}$. This implies the proposition for $c=1$ in the case where $X$ is smooth.

Now consider the general case. Since the problem is local, we can assume that $X$ is analytic in $U=D_{1} \times D_{2}$ where $D_{1}$ and $D_{2}$ are the unit balls in $\mathbb{C}^{p}$ and $\mathbb{C}^{k-p}$ respectively. We can also 
assume that the canonical projection $\pi: D_{1} \times D_{2} \rightarrow D_{1}$ is proper on $X$. Hence, $\pi: X \rightarrow D_{1}$ defines a ramified covering. Let $m$ denote the degree of this covering. For $u \in \mathscr{G}$, define a function $u^{\prime \prime}$ on $D_{1}$ by

$$
u^{\prime \prime}(x):=\sum_{z \in \pi^{-1}(x) \cap X} u(z) .
$$

Since $d d^{c} u^{\prime \prime}=\pi_{*}\left(d d^{c}(u[X])\right) \geq 0, u^{\prime \prime}$ is equal almost everywhere to a psh function $u^{\prime}$. It is easy to check that the family $\mathscr{G}^{\prime}$ of these functions $u^{\prime}$ is compact in $L_{l o c}^{1}\left(D_{1}\right)$. Fix a ball $D$ containing $\pi(K)$ such that $\bar{D} \subset D_{1}$. We need the following Lojasiewicz type inequality, see [18, Proposition 4.11], which implies that $z \mapsto \pi^{-1}(z) \cap X$ is Hölder continuous of exponent $1 / m$ with respect to the Hausdorff metric. The lemma is however more precise and is of independent interest.

Lemma 4.3. - There is a constant $A>0$ such that for $z \in D$ and $x \in X$ with $\pi(x) \in D$, we have

$$
\operatorname{dist}\left(\pi^{-1}(z) \cap X, x\right) \leq A \operatorname{dist}(z, \pi(x))^{1 / m} .
$$

Moreover, if $y$ and $z$ are in $D$ we can write

$$
\pi^{-1}(y) \cap X=\left\{y^{(1)}, \ldots, y^{(m)}\right\} \quad \text { and } \quad \pi^{-1}(z) \cap X=\left\{z^{(1)}, \ldots, z^{(m)}\right\}
$$

so that

$$
\operatorname{dist}\left(y^{(i)}, z^{(i)}\right) \leq A \operatorname{dist}(y, z)^{1 / m} \quad \text { for } 1 \leq i \leq m \text {. }
$$

Proof. - We prove the first assertion. Let $x_{j}, p+1 \leq j \leq k$, denote the last $k-p$ coordinates of $x$. Let $z^{(1)}, \ldots, z^{(m)}$ denote the points in $\pi^{-1}(z) \cap X$ and $z_{p+1}^{(1)}, \ldots, z_{k}^{(1)}, \ldots, z_{p+1}^{(m)}$, $\ldots, z_{k}^{(m)}$ their last $k-p$ coordinates. Here, the points in $\pi^{-1}(z) \cap X$ are repeated according to their multiplicities. For $w \in D_{1}$, define $w^{(i)}$ and $w_{j}^{(i)}$ in the same way. We consider the Weierstrass polynomials on $t \in \mathbb{C}$

$$
\prod_{i=1}^{m}\left(t-w_{j}^{(i)}\right)=t^{m}+a_{j, m-1}(w) t^{m-1}+\cdots+a_{j, 0}(w)=P_{j}(t, w) .
$$

The coefficients of these polynomials are holomorphic with respect to $w \in D_{1}$. The analytic set defined by the polynomials $P_{j}$ contains $X$. In particular, we have $P_{j}\left(x_{j}, \pi(x)\right)=0$. We consider the case where $z \neq \pi(x)$, otherwise the lemma is clear. We will show the existence of a $z^{(i)}$ with good estimates on $z_{j}^{(i)}-x_{j}$.

Fix a constant $c>1$ large enough. There is an integer $2 \leq l \leq 4 m(k-p)+2$ such that $P_{j}(t, \pi(x))$ has no root $t$ with

$$
(l-1) c \sqrt[m]{\|z-\pi(x)\|}<\left|t-x_{j}\right| \leq(l+1) c \sqrt[m]{\|z-\pi(x)\|}
$$

for every $p+1 \leq j \leq k$. We call this the security ring. For $\theta \in \mathbb{R}$ define

$$
\xi_{j}:=l c \sqrt[m]{\|z-\pi(x)\|} e^{i \theta}+x_{j}
$$

and

$$
G_{j, c, \theta}(w):=c^{-m+1} \prod_{i=1}^{m}\left(\xi_{j}-w_{j}^{(i)}\right)=c^{-m+1} P_{j}\left(\xi_{j}, w\right)
$$


Observe that the functions $G_{j, c, \theta}(w)$ are Lipschitz with respect to $w$ in a neighbourhood of $D$ uniformly with respect to $(j, c, \theta)$. Using the choice of $l$, we have

$$
\left|G_{j, c, \theta}(\pi(x))\right|=c^{-m+1}\left|P_{j}\left(\xi_{j}, \pi(x)\right)\right| \geq c\|z-\pi(x)\| .
$$

Hence, if $c$ is large enough, since the $G_{j, c, \theta}(w)$ are uniformly Lipschitz, they do not vanish on the ball $\widetilde{D}$ of center $\pi(x)$ and of radius $2\|z-\pi(x)\|$. Note that here we only need to consider the case where $z$ and $\pi(x)$ are close enough, and we have $\widetilde{D} \Subset D_{1}$. We denote by $\Sigma$ the boundary of the polydisc $H$ of center $\left(x_{p+1}, \ldots, x_{k}\right) \in \mathbb{C}^{k-p}$ and of radius $l c \sqrt[m]{\|z-\pi(x)\|}$. The $P_{j}(t, w)$ have no zero there when $w \in \widetilde{D}$. Then, $X$ does not intersect $\widetilde{D} \times \Sigma$. Since $z \in \widetilde{D}$ and $x \in X$, by continuity, there is a point $z^{(i)}$ satisfying $\left|z_{j}^{(i)}-x_{j}\right| \leq l c \sqrt[m]{\|z-\pi(x)\|}$. This gives the first assertion of the lemma.

We now prove the second assertion. Fix a point $x$ in $\pi^{-1}(y) \cap X$ and use the above construction. In the box $\widetilde{D} \times H, X$ is a ramified covering over $\widetilde{D}$ of some degree $s \leq m$. So we can write with an arbitrary order

$$
\pi^{-1}(y) \cap X \cap \widetilde{D} \times H=\left\{y^{(1)}, \ldots, y^{(s)}\right\} \quad \text { and } \quad \pi^{-1}(z) \cap X \cap \widetilde{D} \times H=\left\{z^{(1)}, \ldots, z^{(s)}\right\}
$$

with the desired estimates on $\left|y^{(i)}-z^{(i)}\right|$, since the diameter of $\widetilde{D} \times H$ is controled by $\|y-z\|^{1 / m}$. This gives a partial correspondence between $\pi^{-1}(y) \cap X$ and $\pi^{-1}(z) \cap X$.

Choose another point $x^{\prime} \in \pi^{-1}(y) \cap X$ outside $\widetilde{D} \times H$ and repeat the construction in order to obtain a box $\widetilde{D} \times H^{\prime}$. We only replace the constant $c$ by $8[m(k-p)+1] c$. This garantees that either $D \times H$ and $D \times H^{\prime}$ are disjoint or $\widetilde{D} \times H$ is contained in $\widetilde{D} \times H^{\prime}$ because of the security rings. In the last situation, we remove the box $\widetilde{D} \times H$. Then, we repeat the construction for points outside the boxes obtained so far. After less than $m$ steps, we obtain a finite family of boxes which induces a complete correspondence between $\pi^{-1}(y) \cap X$ and $\pi^{-1}(z) \cap X$ satisfying the lemma.

Lemma 4.4. - We have $\nu\left(u^{\prime}, x\right)<m^{p} \delta$ for every $u^{\prime} \in \mathscr{G}^{\prime}$ and $x \in D_{1}$.

Proof. - Consider the functions $u^{\prime} \in \mathscr{G}^{\prime}$ and $u^{\prime \prime}$ as above, see (4). Let $y$ be a point in $\pi^{-1}(x) \cap X$ and $V$ a neighbourhood of $y$ such that $\pi^{-1}(x) \cap X \cap V=\{y\}$. We can choose $V$ so that $X \cap V$ is a ramified covering over $\pi(V)$. Let $l$ denote the degree of this covering. Consider the current $R:=d d^{c}(u[X])$ in $V$. In a neighbourhood of $x, d d^{c} u^{\prime}$ (which is equal to $d d^{c} u^{\prime \prime}$ ) is the sum of the currents $\pi_{*}(R)$ for $y$ varying in $\pi^{-1}(x) \cap X$. Since $\nu(R, y)<\delta$ and $l \leq m$, it is enough to prove that $\nu\left(\pi_{*}(R), x\right) \leq l^{p-1} \nu(R, y)$. Assume that $y=0$ and $x=0$ in order to simplify the notation. If $z=\left(z^{\prime}, z^{\prime \prime}\right)=\left(z_{1}, \ldots, z_{p}, z_{p+1}, \ldots, z_{k}\right)$ denote the coordinates in $\mathbb{C}^{k}=\mathbb{C}^{p} \times \mathbb{C}^{k-p}$, then the mass of $\pi_{*}(R) \wedge\left(d d^{c} \log \left\|z^{\prime}\right\|\right)^{p-1}$ at $x=0$ is equal to $\nu\left(\pi_{*}(R), 0\right)$. It follows from the definition of $\pi_{*}$ that the mass of $R \wedge\left(d d^{c} \log \left\|z^{\prime}\right\|\right)^{p-1}$ at $y=0$ is also equal to $\nu\left(\pi_{*}(R), 0\right)$. Define $v:=\max \left(\log \left\|z^{\prime}\right\|, l \log \left\|z^{\prime \prime}\right\|-M\right)$ with $M>0$ large enough. Lemma 4.3 applied to $X \cap V$ implies that $v=\log \left\|z^{\prime}\right\|$ on $X \cap V$. Hence, $R \wedge\left(d d^{c} \log \left\|z^{\prime}\right\|\right)^{p-1}=$ $R \wedge\left(d d^{c} v\right)^{p-1}$. Since $v \geq l \log \|z\|-M^{\prime}, M^{\prime}>0$, the comparison lemma in [6] implies that the mass of $R \wedge\left(d d^{c} v\right)^{p-1}$ at 0 is smaller than the mass of $l^{p-1} R \wedge\left(d d^{c} \log \|z\|\right)^{p-1}$ at 0 which is equal to $l^{p-1} \nu(R, 0)$. This completes the proof.

End of the proof of Proposition 4.2. Now, we apply the case of smooth variety to $\mathscr{G}^{\prime}$. If $0<\rho<1$ then $\sup _{B} u^{\prime} \geq m^{p} \delta \log \rho-$ const, where $B$ is the ball of center $\pi(a)$ and of radius $\rho$ in $\mathbb{C}^{p}$. Let $B^{\prime}$ be the connected component of $X \cap \pi^{-1}(B)$ which contains $a$. This 
is a ramified covering over $B$. Since $u$ is negative, we have $\sup _{B^{\prime}} u \geq \sup _{B} u^{\prime \prime} \geq \sup _{B} u^{\prime}$, see Proposition 2.4(b). Lemma 4.3 implies that $B^{\prime}$ is contained in the union of the balls of center in $\pi^{-1}(\pi(a)) \cap X$ and of radius $A \rho^{1 / m}, A>0$. In this union, consider the connected component containing $a$. It has diameter $\leq 2 m A \rho^{1 / m}$. Hence, $B^{\prime}$ is contained in the ball $B_{a}^{X}(r)$ of center $a$ and of radius $r:=2 m A \rho^{1 / m}$ in $X$. We have

$$
\sup _{B_{a}^{X}(r)} u \geq m^{p} \delta \log \rho-\text { const } \geq m^{p+1} \delta \log r-\text { const }
$$

for $0<\rho<1$. This gives the estimate in the proposition with $c=m^{p+1}$.

Consider the case where $X$ is an analytic subset of pure dimension $p$ of $\mathbb{P}^{k}$. The following proposition is a direct consequence of the last one.

Proposition 4.5. - Let $\mathscr{G} \subset L^{1}(X)$ be a compact family of modulo $T$ wpsh functions on $X$. Let $\delta>0$ such that $\nu_{X}(u, x)<\delta$ for $u \in \mathscr{G}$ and $x \in X$. Then, there exist constants $c>0$ and $A>0$ such that

$$
\sup _{B_{a}^{X}(r)} u \geq c \delta \log r-A \quad \text { for } u \in \mathscr{G}, a \in X \text { and } 0<r<1 .
$$

Moreover, the constant $c$ is independent of $\mathscr{G}$ and of $\delta$.

The following result is a consequence of an inequality due to Demailly and Méo [6, 26]. It gives a bound for the volume of the set where the Lelong numbers are large.

Lemma 4.6. - Let u be a modulo $T$ wpsh function on an analytic set $X$ of pure dimension $p$ in $\mathbb{P}^{k}$. Let $\beta \geq 0$ be a constant and $q$ the dimension of $\left\{\nu_{X}(u, x)>\beta\right\}$. Consider a finite family of analytic sets $Z_{r}, 1 \leq r \leq s$, of pure dimension $q$ in $X$. Assume that $\nu_{X}(u, x) \geq \nu_{r}$ for $x \in Z_{r}$ where $\left(\nu_{r}\right)$ is a decreasing sequence such that $\nu_{r} \geq 2 \beta$. Assume also that $\operatorname{deg} Z_{r} \geq d_{r}$ where the $d_{r}$ 's are positive and satisfy $d_{r-1} \leq \frac{1}{2} d_{r}$. Then

$$
\sum_{r} d_{r} \nu_{r}^{p-q} \leq 2^{p-q+1} \operatorname{deg}(X)^{p-q} .
$$

Proof. - Define $R:=d d^{c}(u[X])+T \wedge[X]$, then $R$ is of bidimension $(p-1, p-1)$. Recall that $\nu_{X}(u, x)=\nu(R, x)$. The mass of $R$ is equal to $\operatorname{deg}(X)$. Define $Z_{1}^{\prime}:=Z_{1}$ and for $r \geq 2$, $Z_{r}^{\prime}$ the union of irreducible components of $Z_{r}$ which are not components of $Z_{1} \cup \cdots \cup Z_{r-1}$. So, $Z_{i}^{\prime}$ and $Z_{r}^{\prime}$ have no common component for $i \neq r$. Let $d_{r}^{\prime}$ denote the degree of $Z_{r}^{\prime}$. We have $d_{1}^{\prime}+\cdots+d_{r}^{\prime} \geq d_{r}$ for $r \geq 1$. We also have $\nu(R, x) \geq \nu_{r}$ on $Z_{r}^{\prime}$. The inequality of Demailly-Méo [6, 26] implies that

$$
\sum_{r}\left(\operatorname{deg} Z_{r}^{\prime}\right)\left(\nu_{r}-\beta\right)^{p-q} \leq\|R\|^{p-q}=(\operatorname{deg} X)^{p-q} .
$$

Hence, since $\beta \leq \nu_{r} / 2$,

$$
\sum_{r} d_{r}^{\prime} \nu_{r}^{p-q} \leq 2^{p-q}(\operatorname{deg} X)^{p-q}
$$

$4{ }^{\mathrm{e}}$ SÉRIE - TOME $41-2008-\mathrm{N}^{\mathrm{o}} 2$ 
On the other hand, using the properties of $d_{r}, d_{r}^{\prime}$, the fact that $\left(\nu_{r}\right)$ is decreasing and the Abel's transform, we obtain

$$
\begin{aligned}
\sum_{r} d_{r}^{\prime} \nu_{r}^{p-q}= & d_{1}^{\prime}\left(\nu_{1}^{p-q}-\nu_{2}^{p-q}\right)+\left(d_{1}^{\prime}+d_{2}^{\prime}\right)\left(\nu_{2}^{p-q}-\nu_{3}^{p-q}\right)+\cdots+ \\
& +\left(d_{1}^{\prime}+\cdots+d_{s-1}^{\prime}\right)\left(\nu_{s-1}^{p-q}-\nu_{s}^{p-q}\right)+\left(d_{1}^{\prime}+\cdots+d_{s}^{\prime}\right) \nu_{s}^{p-q} \\
\geq & d_{1}\left(\nu_{1}^{p-q}-\nu_{2}^{p-q}\right)+\cdots+d_{s-1}\left(\nu_{s-1}^{p-q}-\nu_{s}^{p-q}\right)+d_{s} \nu_{s}^{p-q} \\
\geq & \frac{1}{2} d_{1} \nu_{1}^{p-q}+\cdots+\frac{1}{2} d_{s} \nu_{s}^{p-q} .
\end{aligned}
$$

This proves the lemma.

\section{Asymptotic contraction}

In this section, we study the speed of contraction of $f^{n}$. More precisely, we want to estimate the size of the largest ball contained in the image of a fixed ball by $f^{n}$. Our main result is the following theorem where the balls in $X$ are defined in Section 4.

THeORem 5.1. - Let $f$ be a holomorphic endomorphism of algebraic degree $d \geq 2$ of $\mathbb{P}^{k}$ and $X$ an analytic subset of pure dimension $p, 1 \leq p \leq k$, invariant by $f$, i.e. $f(X)=X$. There exists a constant $c>0$ such that if $B$ is a ball of radius $r$ in $X$ with $0<r<1$, then for every $n \geq 0, f^{n}(B)$ contains a ball in $X$ of radius $\exp \left(-c r^{-2 p} d^{n}\right)$.

COROLlary 5.2. - Let $f$ be a holomorphic endomorphism of algebraic degree $d \geq 2$ of $\mathbb{P}^{k}$. There exists a constant $c>0$ such that if $B$ is a ball of radius $r$ in $\mathbb{P}^{k}$ with $0<r<1$, then $f^{n}(B)$ contains a ball of radius $\exp \left(-c r^{-2 k} d^{n}\right)$ for every $n \geq 0$.

Let $H$ be a hypersurface in $\mathbb{P}^{k}$ which does not contain any irreducible component of $X$ such that the restriction of $f$ to $X \backslash H$ is of maximal rank at every point. We choose $H$ containing $\operatorname{sing}(X) \cup f^{-1}(\operatorname{sing}(X))$. If $\delta$ is the degree of $H$, there is a negative function $u$ on $\mathbb{P}^{k}$ psh modulo $T$ such that $d d^{c} u=\delta^{-1}[H]-T$.

Lemma 5.3. - There are positive constants $c_{1}$ and $c_{2}$ such that if $B$ is a ball of center a and of radius $0<r<1$ in $X$ then $f(B)$ contains the ball of center $f(a)$ and of radius $c_{1} r \exp \left(c_{2} u(a)\right)$ in $X$. Moreover, if $u(a) \neq-\infty$ then the differential at $f(a)$ of $f^{-1}$ restricted to $X$ satisfies $\left\|D f_{\mid X}^{-1}(f(a))\right\| \leq c_{1}^{-1} \exp \left(-c_{2} u(a)\right)$.

Proof. - The constants $c_{i}$ that we use here are independent of $a$ and $r$. We only have to consider the case where $u(a) \neq-\infty$. Observe that when $c_{1}$ is small and $c_{2}$ is large enough, the ball of center $f(a)$ and of radius $c_{1} r \exp \left(c_{2} u(a)\right)$ does not intersect $H$ which contains $\operatorname{sing}(X)$. Let $\pi: \widetilde{X} \rightarrow X \subset \mathbb{P}^{k}$ be a desingularization of $X$ and $A:=\|\pi\|_{\mathscr{C}^{1}}$. If $\pi(\widetilde{a})=a$, and if $\widetilde{B}$ is the ball of center $\widetilde{a}$ and of radius $\widetilde{r}:=A^{-1} r$ then $\pi(\widetilde{B})$ is contained in the ball $B$. Define $h:=f \circ \pi, \widetilde{u}:=u \circ \pi$ and $\widetilde{T}:=\pi^{*}(T)$. Since $T$ has continuous local potentials, so does $\pi^{*}(T)$.

The current $\pi^{*}[H]$ is supported in $\pi^{-1}(H)$ and satisfies $d d^{c} \widetilde{u}=\delta^{-1} \pi^{*}[H]-\pi^{*}(T)$. Since $\widetilde{u}=-\infty$ exactly on $\pi^{-1}(H)$ and since $\pi^{*}(T)$ has continuous local potentials, the support of $\pi^{*}[H]$ is exactly $\pi^{-1}(H)$. So, $\pi^{*}[H]$ is a combination with strictly positive coefficients of the currents of integration on irreducible components of $\pi^{-1}(H)$. Observe that $h$ is of maximal 
rank outside $\pi^{-1}(H)$. It is enough to prove that $h(\widetilde{B})$ contains the ball of center $h(\widetilde{a})$ and of radius $c_{1} r \exp \left(c_{2} \widetilde{u}(\widetilde{a})\right)$ in $X$.

We can assume that $r$ is small and works in the local setting. We use holomorphic coordinates $x=\left(x_{1}, \ldots, x_{p}\right)$ of $\widetilde{X}$ and $y=\left(y_{1}, \ldots, y_{k}\right)$ of $\mathbb{P}^{k}$ in small neighbourhoods $W$ and $U$ of $\tilde{a}$ and $a$ respectively. Write $h=\left(h_{1}, \ldots, h_{k}\right)$ and consider a holomorphic function $\varphi$ on $W$ such that $\varphi^{-1}(0)=\pi^{-1}(H) \cap W$. Then, $\delta^{-1} \pi^{*}[H] \geq \varepsilon d d^{c} \log |\varphi|$ with $\varepsilon>0$ small enough. We have $d d^{c}(\widetilde{u}-\varepsilon \log |\varphi|) \geq-\widetilde{T}$. It follows that $\widetilde{u}-\varepsilon \log |\varphi|$ is a difference of a psh function and a potential of $\widetilde{T}$. Since $\widetilde{T}$ has local continuous potentials, $\widetilde{u}-\varepsilon \log |\varphi|$ is bounded from above. Up to multiplying $\varphi$ by a constant, we can assume that $\varepsilon \log |\varphi| \geq \widetilde{u}$.

If $J \subset\{1, \ldots, k\}$ is a multi-index of length $p$, denote by $M_{J}$ the matrix $\left(\partial h_{j} / \partial x_{i}\right)$ with $1 \leq$ $i \leq p$ and $j \in J$. Since $h$ is of maximal rank outside $\pi^{-1}(H)$, the zero set of $\sum_{J}\left|\operatorname{det} M_{J}\right|^{2}$ is contained in $\{\varphi=0\}$. The Lojasiewicz's inequality [31] implies that $\sum_{J}\left|\operatorname{det} M_{J}\right|^{2} \geq$ $c_{3}|\varphi|^{c_{4}}$ for some constants $c_{3}>0$ and $c_{4}>0$. Up to a permutation of the coordinates $y$, we can assume that $|\operatorname{det} M(\widetilde{a})| \geq c_{5}|\varphi(\widetilde{a})|^{c_{2} \varepsilon / 2} \geq c_{5} \exp \left(c_{2} \widetilde{u}(\widetilde{a}) / 2\right)$ where $c_{2}, c_{5}$ are positive constants and $M$ is the matrix $\left(\partial h_{j} / \partial x_{i}\right)_{1 \leq i, j \leq p}$. Define $h^{\prime}:=\left(h_{1}, \ldots, h_{p}\right)$. The precise version of the implicit function theorem [31, p.106] implies that $h^{\prime}$ defines a bijection from an open subset of $\widetilde{B}$ to a ball of center $h^{\prime}(\widetilde{a})$ and of radius $c_{6} \widetilde{r}|\operatorname{det} M(\widetilde{a})|^{2}, c_{6}>0$. This proves the first assertion in the lemma. For the second one, we have $\left\|D h^{-1}\right\| \lesssim|\operatorname{det} M(\widetilde{a})|^{-1}$ at $h^{\prime}(\widetilde{a})$ which gives the result.

Proof of Theorem 5.1. By Corollary 3.9, the sequence of functions $\left(d^{-n} u \circ f^{n}\right)$ is bounded in $L^{1}(X)$. Since

$$
d^{-n}\left(u+u \circ f+\cdots+u \circ f^{n-1}\right)=\sum_{i=0}^{n-1} d^{-(n-i)}\left(d^{-i} u \circ f^{i}\right),
$$

the $L^{1}(X)$-norm of $d^{-n}\left(u+u \circ f+\cdots+u \circ f^{n-1}\right)$ is bounded by a constant $c^{\prime}>0$ independent of $n$. If $A>0$ is a constant large enough, the set of points $x \in X$ satisfying

$$
u(x)+u \circ f(x)+\cdots+u \circ f^{n-1}(x) \leq-A r^{-2 p} d^{n}
$$

has Lebesgue measure $\leq c^{\prime} A^{-1} r^{2 p}$. By a theorem of Lelong [23, 6], the volume of a ball of radius $r / 2$ in $X$ is $\geq c^{\prime \prime} r^{2 p}, c^{\prime \prime}>0$. Therefore, since $A$ is large, there is a point $b \in X$, depending on $n$, such that $|b-a| \leq r / 2$ and

$$
u(b)+u \circ f(b)+\cdots+u \circ f^{n-1}(b) \geq-A r^{-2 p} d^{n} .
$$

Lemma 5.3 applied inductively to balls centered at $f^{i}(b)$ implies that $f^{n}(B)$ contains the ball of center $f^{n}(b)$ of radius

$$
\frac{1}{2} c_{1}^{n} r \exp \left(c_{2} u(b)+\cdots+c_{2} u\left(f^{n-1}(b)\right)\right) .
$$

We obtain the result using (5) and the estimate $\frac{1}{2} c_{1}^{n} r \geq \exp \left(-c_{3} r^{-2 p} d^{n}\right)$ for $0<r<1$, where $c_{3}>0$ is a constant.

ReMARK 5.4. - With the same argument we also get the following. Let $B_{x}$ denote the ball of center $x$ and of radius $0<r<1$ in $X$. Let $r_{n}(x)$ be the maximal radius of the ball 
centered at $f^{n}(x)$ and contained in $f^{n}\left(B_{x}\right)$. Then, there is a constant $A>0$ such that

$$
\frac{\log r_{n}(x)}{d^{n}} \geq-\frac{A(n+1)-\log r}{d^{n}}+\frac{c_{2}}{d^{n}} \sum_{i=0}^{n-1} u \circ f^{i}(x) .
$$

Consequently, there is a constant $c>0$ such that

$$
\int_{X} \frac{\log r_{n}(x)}{d^{n}} \omega^{p} \geq-c+\frac{\log r}{d^{n}} \operatorname{deg} X
$$

We can also replace $\omega^{p}$ by any PB measure on $X$, i.e. a measure such that modulo $T$ wpsh functions are integrable, see [10].

In the following result, we use the Lebesgue measure $\operatorname{vol}_{X}$ on $X$ induced by the FubiniStudy form restricted to $X$.

Theorem 5.5. - Let $f$ and $X$ be as in Theorem 5.1. Let $Z$ be a Borel set in $X$ and $n \geq 0$. Then there is a Borel set $Z_{n} \subset Z$ with $\operatorname{vol}_{X}\left(Z_{n}\right) \geq \frac{1}{2} \operatorname{vol}_{X}(Z)$ such that the restriction $f_{\mid X}^{n}$ of $f^{n}$ to $X$ defines a locally bi-Lipschitz map from $Z_{n}$ to $f^{n}\left(Z_{n}\right)$. Moreover, the differential of the inverse map $f_{\mid X}^{-n}$ satisfies $\left\|D f_{\mid X}^{-n}\right\| \leq \exp \left(c \operatorname{vol}(Z)^{-1} d^{n}\right)$ on $f^{n}\left(Z_{n}\right)$ with a constant $c>0$ independent of $n$ and $Z$. In particular, we have $\operatorname{vol}_{X}\left(f^{n}(Z)\right) \geq \exp \left(-c^{\prime} \operatorname{vol}_{X}(Z)^{-1} d^{n}\right)$ for some constant $c^{\prime}>0$ independent of $n$ and $Z$.

Proof. - As in (5), there is a subset $Z_{n}$ of $Z$ with $\operatorname{vol}_{X}\left(Z_{n}\right) \geq \frac{1}{2} \operatorname{vol}_{X}(Z)$ such that

$$
u(b)+u \circ f(b)+\cdots+u \circ f^{n-1}(b) \geq-A \operatorname{vol}_{X}(Z)^{-1} d^{n}
$$

for $b \in Z_{n}$, where $A>0$ is a fixed constant large enough. In particular, we have $u \circ f^{i}(b) \neq$ $-\infty$ for $i \leq n-1$. It follows from the definition of $u$ that $f_{\mid X}^{n}$ defines a bijection between a neighbourhood of $b$ and a neighbourhood of $f^{n}(b)$ in $X$. Hence, $f_{\mid X}^{n}: Z_{n} \rightarrow f^{n}\left(Z_{n}\right)$ is locally bi-Lipschitz. Applying Lemma 5.3 inductively gives the estimate on $\left\|D f_{\mid X}^{-n}\right\|$ at $f^{n}(b)$.

Since the fibers of $f^{n}$ contain at most $d^{k n}$ points, the estimate on $\left\|D f_{\mid X}^{-n}\right\|$ implies

$$
\operatorname{vol}_{X}\left(f^{n}(Z)\right) \geq \operatorname{vol}_{X}\left(f^{n}\left(Z_{n}\right)\right) \gtrsim d^{-k n} \operatorname{vol}_{X}(Z) \exp \left(-c \operatorname{vol}_{X}(Z)^{-1} d^{n}\right)^{2 p} .
$$

The last assertion in the theorem follows.

REMARK 5.6. - It is not difficult to extend Theorems 5.1 and 5.5 to the case of meromorphic maps or correspondences on compact Kähler manifolds. We can use the continuity of $f^{*}$ on the space DSH in order to estimate the $L^{1}$-norm of $u \circ f^{n}$ for $u \in \mathrm{DSH}$, see [10]. The volume estimate in Theorem 5.5 for meromorphic maps on smooth manifolds was obtained in [21], see also [16, 13, 20] for earlier versions.

Let $\mathscr{G}$ be a compact family of modulo $T$ wpsh functions on $X$. Let $\mathscr{H}_{n}$ denote the family of $T$ wpsh functions which are equal almost everywhere to $d^{-n} u \circ f^{n}, u \in \mathscr{G}$. Define

$$
\nu_{n}:=\sup \left\{\nu_{X}(u, a), u \in \mathscr{H}_{n}, a \in X\right\} .
$$

We have the following result.

Proposition 5.7. - Assume that $\inf \nu_{n}=0$. Then, $d^{-n} u_{n} \circ f^{n} \rightarrow 0$ in $L^{1}(X)$ for all $u_{n} \in \mathscr{G}$. In particular, the hypothesis is satisfied when there is an increasing sequence $\left(n_{i}\right)$ such that $d^{-n_{i}} u_{n_{i}} \circ f^{n_{i}}$ converge to 0 in $L^{1}(X)$ for all $u_{n_{i}} \in \mathscr{G}$. 
Proof. - Consider a sequence $\left(d^{-n_{i}} u_{n_{i}} \circ f^{n_{i}}\right)$ converging in $L^{1}(X)$ to a modulo $T$ wpsh function $u$. Corollary 3.9 implies that $u \leq 0$. We want to prove that $u=0$. If not, since $u$ is upper semi-continuous, there is a constant $\alpha>0$ such that $u \leq-2 \alpha$ on some ball $B$ of radius $0<r<1$ in $X$. By Lemmas 3.4 and 3.8, for $i$ large enough, we have $d^{-n_{i}} u_{n_{i}} \circ f^{n_{i}} \leq-\alpha$ almost everywhere on $B$.

Fix $\delta>0$ small enough and $m$ such that $\nu_{m}<\delta$. Consider only the $n_{i}$ larger than $m$. Then, $d^{-m} u_{n_{i}} \circ f^{m} \leq-d^{n_{i}-m} \alpha$ almost everywhere on $f^{n_{i}-m}(B)$. By Theorem 5.1, $f^{n_{i}-m}(B)$ contains a ball $B_{i}$ of radius $\exp \left(-c r^{-2 p} d^{n_{i}-m}\right)$ in $X$ with $c>1$. If $v_{i} \in \mathscr{H}_{m}$ is equal almost everywhere to $d^{-m} u_{n_{i}} \circ f^{m}$, then $v_{i} \leq-d^{n_{i}-m} \alpha$ almost everywhere on $B_{i}$. It follows from Proposition 3.2(b) that this inequality holds everywhere on $B_{i}$. By Proposition 4.5, there are a constant $c^{\prime}>0$ independent of $\mathscr{G}, r, \delta, m$, and a constant $A>0$ such that

$$
-c^{\prime} \delta r^{-2 p} d^{n_{i}-m}-A \leq \sup _{B_{i}} v_{i} \leq-d^{n_{i}-m} \alpha .
$$

This is a contradiction if $\delta$ is chosen small enough and if $n_{i}$ is large enough.

Assume now that $d^{-n_{i}} u_{n_{i}} \circ f^{n_{i}}$ converge to 0 in $L^{1}(X)$ for all $u_{n_{i}} \in \mathscr{G}$. Then, for every $\varepsilon>0$, we have $\nu(u, a)<\varepsilon$ for $u \in \mathscr{H}_{n_{i}}, a \in X$ and for $i$ large enough. Therefore, $\inf \nu_{n}=0$. Here, we use that if positive closed currents $R_{n}$ converge to $R$ and $a_{n} \rightarrow a$ then $\lim \sup \nu\left(R_{n}, a_{n}\right) \leq \nu(R, a)$.

Corollary 5.8. - Let $\mathscr{F}$ be a family of positive closed $(1,1)$-currents of mass 1 on $\mathbb{P}^{k}$. Assume that there is an increasing sequence of integers $\left(n_{i}\right)$ such that $d^{-n_{i}}\left(f^{n_{i}}\right)^{*}\left(S_{n_{i}}\right) \rightarrow T$ for all $S_{n_{i}} \in \mathscr{F}$. Then, $d^{-n}\left(f^{n}\right)^{*}\left(S_{n}\right) \rightarrow T$ for all $S_{n} \in \mathscr{F}$.

Proof. - Observe that the hypothesis implies that $d^{-n_{i}}\left(f^{n_{i}}\right)^{*}\left(S_{n_{i}}\right) \rightarrow T$ for all $S_{n_{i}} \in \overline{\mathscr{F}}$. So, we can replace $\mathscr{F}$ by $\overline{\mathscr{F}}$ and assume that $\mathscr{F}$ is compact. To each current $S \in \mathscr{F}$ we associate a modulo $T$ psh function $u$ on $\mathbb{P}^{k}$ such that $d d^{c} u=S-T$. Subtracting from $u$ some constant allows us to have $\max _{\mathbb{P} k} u=0$. Proposition 3.3 and Lemma 3.4 imply that the family $\mathscr{G}$ of these functions $u$ is compact. The hypothesis and Corollary 3.9 imply that $d^{-n_{i}} u_{n_{i}} \circ f^{n_{i}} \rightarrow 0$ for $u_{n_{i}} \in \mathscr{G}$. Proposition 5.7 gives the result.

COROLlaRY 5.9. - Let $\mathscr{F}$ be a compact family of positive closed $(1,1)$-currents of mass 1 on $\mathbb{P}^{k}$. Assume that for any $S \in \mathscr{F}$, the Lelong number of $S$ vanishes at every point out of $\operatorname{supp}(\mu)$. Then, $d^{-n}\left(f^{n}\right)^{*}\left(S_{n}\right) \rightarrow T$ for any sequence $\left(S_{n}\right) \subset \mathscr{F}$.

Proof. - Let $\mathscr{G}$ and $\mathscr{H}_{n}$ be defined as above. Define also

$$
\nu_{n}^{\prime}:=\sup \left\{\nu_{X}(u, a), u \in \mathscr{H}_{n}, a \in \operatorname{supp}(\mu)\right\}
$$

and

$$
\nu_{n}^{\prime \prime}:=\sup \left\{\nu_{X}(u, a), u \in \mathscr{H}_{n}, a \notin \operatorname{supp}(\mu)\right\} .
$$

Corollary 3.9 implies that $\lim \nu_{n}^{\prime}=0$. On the other hand, by hypothesis, $\nu_{0}^{\prime \prime}=0$. Since $\mathbb{P}^{k} \backslash \operatorname{supp}(\mu)$ is totally invariant, Proposition 4.1, applied to $X=\mathbb{P}^{k}$, implies that $\nu_{n}^{\prime \prime}=0$ for every $n$. Hence, $\nu_{n}=\nu_{n}^{\prime}$ and $\nu_{n} \rightarrow 0$. We apply Proposition 5.7 in order to conclude. Note that the corollary still holds if we only assume that $\inf \nu_{n}^{\prime \prime}=0$.

We prove as in Proposition 5.7 the following lemma. 
Lemma 5.10. - Let $\left(u_{n_{i}}\right)$ be a sequence of modulo $T$ wpsh functions on $X$, bounded in $L^{1}(X)$. Assume that $d^{-n_{i}} u_{n_{i}} \circ f^{n_{i}}$ converge to a modulo $T$ wpsh function $v$. Assume also that for every $\delta>0$, there is a subsequence $\left(u_{m_{i}}\right) \subset\left(u_{n_{i}}\right)$ converging to a modulo $T$ wpsh function $w$ with $\nu_{X}(w, a)<\delta$ at every point $a \in X$. Then, $v=0$.

Proof. - Corollary 3.9 implies that $v \leq 0$. Assume that $v \neq 0$. Then, since $v$ is upper semi-continuous, there is a constant $\alpha>0$ such that $v<-2 \alpha$ on a ball of radius $0<r<1$ on $X$. As in Proposition 5.7, for $i$ large enough we have $u_{n_{i}}<-d^{n_{i}} \alpha$ on a ball $B_{n_{i}}$ of radius $\exp \left(-c r^{-2 p} d^{n_{i}}\right)$ in $X$ with $c>1$.

Fix $\delta>0$ small enough, and $\left(u_{m_{i}}\right)$ and $w$ as above. The property of $w$ implies that if $s$ is an integer large enough, we have $\nu_{X}\left(u_{m_{i}}, a\right)<\delta$ for every $a \in X$ and for $i \geq s$. By Proposition 4.5 applied to the compact family $\left\{u_{m_{i}}, i \geq s\right\} \cup\{w\}$, there is a constant $c^{\prime}>0$ independent of $\delta, r$ and a constant $A>0$ such that

$$
-c^{\prime} \delta r^{-2 p} d^{m_{i}}-A \leq \sup _{B_{m_{i}}} u_{m_{i}} \leq-d^{m_{i}} \alpha \quad \text { for } i \geq s .
$$

This is a contradiction for $m_{i}$ large enough, since $\delta$ is chosen small.

\section{Exceptional sets}

Let $X$ be an analytic subset of pure dimension $p$ in $\mathbb{P}^{k}$ invariant by $f$, i.e. $f(X)=X$. Let $g: X \rightarrow X$ denote the restriction of $f$ to $X$. We will follow the idea of [9] in order to define and study the exceptional analytic subset $\mathscr{E}_{X}$ of $X$ which is totally invariant by $g$, see also $[7,8]$. The following result can be deduced from Section 3.4 in [9].

From now on, each point $x \in X$ is associated to an irreducible component of the germ $(X, x)$. In other words, if $(X, x)$ has $s$ components, one considers $x$ as $s$ different points. The reader can use the lift of $g$ to the normalization of $X$, but for simplicity we keep the notation $g$.

Theorem 6.1. - There is a (possibly empty) proper analytic subset $\mathscr{E}_{X}$ of $X$ which is totally invariant by $g$ and is maximal in the following sense. If $E$ is an analytic subset of dimension $<$ pof $X$ such that $g^{-s}(E) \subset E$ for some $s \geq 1$, then $E \subset \mathscr{E}_{X}$. In particular, there is a maximal proper analytic subset $\mathscr{E}_{\mathbb{P}^{k}}$ of $\mathbb{P}^{k}$ which is totally invariant by $f$.

We will need some precise properties of $\mathscr{E}_{X}$. So, for the reader's convenience, we recall here the construction of $\mathscr{E}_{X}$ and the proof of the previous theorem since the emphasis in [9] is on polynomial-like maps. Observe that $g$ permutes the irreducible components of $X$. Let $m \geq 1$ be an integer such that $g^{m}$ fixes the components of $X$.

Lemma 6.2. - The topological degree of $g^{m}$ is equal to $d^{m p}$, that is, $g^{m}: X \rightarrow X$ defines a ramified covering of degree $d^{m p}$. In particular, for every $x \in X, g^{-m}(x)$ contains at most $d^{m p}$ points and there is a hypersurface $Y$ of $X$ containing $\operatorname{sing}(X) \cup g^{m}(\operatorname{sing}(X))$ such that for $x \in X \backslash Y, g^{-m}(x)$ contains exactly $d^{m p}$ points. 
Proof. - We can work with each component. So, we can assume that $X$ is irreducible. It follows that $g^{m}$ defines a ramified covering. We want to prove that the degree $\delta$ of this covering is equal to $d^{m p}$. Consider the positive measure $\left(f^{m}\right)^{*}\left(\omega^{p}\right) \wedge[X]$. Its mass is equal to $d^{m p} \operatorname{deg}(X)$ since $\left(f^{m}\right)^{*}\left(\omega^{p}\right)$ is cohomologous to $d^{m p} \omega^{p}$. The operator $\left(f^{m}\right)_{*}$ preserves the mass of positive measures. We also have $\left(f^{m}\right)_{*}[X]=\delta[X]$. Hence,

$$
\begin{aligned}
d^{m p} \operatorname{deg}(X) & =\left\|\left(f^{m}\right)^{*}\left(\omega^{p}\right) \wedge[X]\right\|=\left\|\left(f^{m}\right)_{*}\left(\left(f^{m}\right)^{*}\left(\omega^{p}\right) \wedge[X]\right)\right\| \\
& =\left\|\omega^{p} \wedge\left(f^{m}\right)_{*}[X]\right\|=\delta\left\|\omega^{p} \wedge[X]\right\|=\delta \operatorname{deg}(X) .
\end{aligned}
$$

Therefore, $\delta=d^{m p}$. So, we can take for $Y$, a hypersurface containing the ramification values of $f^{m}$ and $\operatorname{sing}(X) \cup g^{m}(\operatorname{sing}(X))$.

Let $Y$ be as above. Observe that if $g^{m}(x) \notin Y$ then $g^{m}$ defines a biholomorphic map between a neighbourhood of $x$ and a neighbourhood of $g^{m}(x)$ in $X$. Let $[Y]$ denote the $(k-$ $p+1, k-p+1)$-current of integration on $Y$ in $\mathbb{P}^{k}$. Since $\left(f^{m n}\right)_{*}[Y]$ is a positive closed $(k-p+1, k-p+1)$-current of mass $d^{m n(p-1)} \operatorname{deg}(Y)$, we can define the following ramification current

$$
R=\sum_{n \geq 0} R_{n}:=\sum_{n \geq 0} d^{-m n p}\left(f^{m n}\right)_{*}[Y] .
$$

By a theorem of Siu [30, 6], for $c>0$, the level set $E_{c}:=\{\nu(R, x) \geq c\}$ of the Lelong number is an analytic set of dimension $\leq p-1$ contained in $X$. Observe that $E_{1}$ contains $Y$. We will see that $R$ is the obstruction for the construction of "regular" orbits.

For any point $x \in X$ let $\lambda_{n}^{\prime}(x)$ denote the number of distinct orbits

$$
x_{-n}, x_{-n+1}, \ldots, x_{-1}, x_{0}
$$

such that $g^{m}\left(x_{-i-1}\right)=x_{-i}, x_{0}=x$ and $x_{-i} \in X \backslash Y$ for $0 \leq i \leq n-1$. These are the "good" orbits. Define $\lambda_{n}:=d^{-m p n} \lambda_{n}^{\prime}$. The function $\lambda_{n}$ is lower semi-continuous with respect to the Zariski topology on $X$. Moreover, by Lemma 6.2, we have $0 \leq \lambda_{n} \leq 1$ and $\lambda_{n}=1$ out of the analytic set $\cup_{i=0}^{n-1} g^{m i}(Y)$. The sequence $\left(\lambda_{n}\right)$ decreases to a function $\lambda$, which represents the asymptotic proportion of orbits in $X \backslash Y$.

Lemma 6.3. - There is a constant $\gamma>0$ such that $\lambda \geq \gamma$ on $X \backslash E_{1}$.

Proof. - We deduce from the Siu's theorem, the existence of a constant $0<\gamma<1$ satisfying $\{\nu(R, x)>1-\gamma\}=E_{1}$. Consider a point $x \in X \backslash E_{1}$. We have $x \notin Y$. Define $\nu_{n}:=\nu\left(R_{n}, x\right)$. We have $\sum \nu_{n} \leq 1-\gamma$. Since $E_{1}$ contains $Y, \nu_{0}=0$ and $F_{1}:=g^{-m}(x)$ contains exactly $d^{m p}$ points. The definition of $\nu_{1}$ implies that $g^{-m}(x)$ contains at most $\nu_{1} d^{m p}$ points in $Y$. Then

$$
\# g^{-m}\left(F_{1} \backslash Y\right)=d^{m p} \#\left(F_{1} \backslash Y\right) \geq\left(1-\nu_{1}\right) d^{2 m p} .
$$

Define $F_{2}:=g^{-m}\left(F_{1} \backslash Y\right)$. The definition of $\nu_{2}$ implies that $F_{2}$ contains at most $\nu_{2} d^{2 m p}$ points in $Y$. Hence, $F_{3}:=g^{-m}\left(F_{2} \backslash Y\right)$ contains at least $\left(1-\nu_{1}-\nu_{2}\right) d^{3 m p}$ points. In the same way, we define $F_{4}, \ldots, F_{n}$ with $\# F_{n} \geq\left(1-\sum \nu_{i}\right) d^{m p n}$. Hence, for every $n$ we get the following estimate:

$$
\lambda_{n}(x) \geq d^{-m p n} \# F_{n} \geq 1-\sum \nu_{i} \geq \gamma
$$

This proves the lemma. 
End of the proof of Theorem 6.1. Let $\mathscr{E}_{X}^{n}$ denote the set of $x \in X$ such that $g^{-m l}(x) \subset E_{1}$ for $0 \leq l \leq n$ and define $\mathscr{E}_{X}:=\cap_{n \geq 0} \mathscr{E}_{X}$. Then, $\left(\mathscr{E}_{X}^{n}\right)$ is a decreasing sequence of analytic subsets of $E_{1}$. It should be stationary. So, there is $n_{0} \geq 0$ such that $\mathscr{E}_{X} n=\mathscr{E}_{X}$ for $n \geq n_{0}$.

By definition, $\mathscr{E}_{X}$ is the set of $x \in X$ such that $g^{-m n}(x) \subset E_{1}$ for every $n \geq 0$. Hence, $g^{-m}\left(\mathscr{E}_{X}\right) \subset \mathscr{E}_{X}$. It follows that the sequence of analytic sets $g^{-m n}\left(\mathscr{E}_{X}\right)$ is decreasing and there is $n \geq 0$ such that $g^{-m(n+1)}\left(\mathscr{E}_{X}\right)=g^{-m n}\left(\mathscr{E}_{X}\right)$. Since $g^{m n}$ is surjective, we deduce that $g^{-m}\left(\mathscr{E}_{X}\right)=\mathscr{E}_{X}$ and hence $\mathscr{E}_{X}=g^{m}\left(\mathscr{E}_{X}\right)$.

Assume as in the theorem that $E$ is analytic with $g^{-s}(E) \subset E$. Define $E^{\prime}:=g^{-s+1}(E) \cup$ $\cdots \cup E$. We have $g^{-1}\left(E^{\prime}\right) \subset E^{\prime}$ which implies $g^{-n-1}\left(E^{\prime}\right) \subset g^{-n}\left(E^{\prime}\right)$ for every $n \geq 0$. Hence, $g^{-n-1}\left(E^{\prime}\right)=g^{-n}\left(E^{\prime}\right)$ for $n$ large enough. This and the surjectivity of $g$ imply that $g^{-1}\left(E^{\prime}\right)=g\left(E^{\prime}\right)=E^{\prime}$. By Lemma 6.2, the topological degree of $\left(g^{m^{\prime}}\right)_{\mid E^{\prime}}$ is at most $d^{m^{\prime}(p-1)}$ for $m^{\prime} \geq 1$. This, the identity $g^{-1}\left(E^{\prime}\right)=g\left(E^{\prime}\right)=E^{\prime}$ together with Lemma 6.3 imply that $E^{\prime} \subset E_{1}$. Hence, $E^{\prime} \subset \mathscr{E}_{X}$ and $E \subset \mathscr{E}_{X}$.

Define $\mathscr{E}_{X}^{\prime}:=g^{-m+1}\left(\mathscr{E}_{X}\right) \cup \cdots \cup \mathscr{E}_{X}$. We have $g^{-1}\left(\mathscr{E}_{X}^{\prime}\right)=g\left(\mathscr{E}_{X}^{\prime}\right)=\mathscr{E}_{X}^{\prime}$. Applying the previous assertion to $E:=\mathscr{E}_{X}^{\prime}$ yields $\mathscr{E}_{X}^{\prime} \subset \mathscr{E}_{X}$. Therefore, $\mathscr{E}_{X}^{\prime}=\mathscr{E}_{X}$ and $g^{-1}\left(\mathscr{E}_{X}\right)=$ $g\left(\mathscr{E}_{X}\right)=\mathscr{E}_{X}$

Remark 6.4. - The maximality of $\mathscr{E}_{X}$ in Theorem 6.1 implies that it does not depend on the choice of $m$ and of the analytic set $Y$ satisfying Lemma 6.2. Moreover, $\mathscr{E}_{X}$ is also the exceptional set associated to $g^{n}$ for every $n \geq 1$. An analytic set, totally invariant by $g^{n}$, is not necessarily totally invariant by $g$, but it is a union of components of such sets. We deduce from our construction that $\mathscr{E}_{\mathbb{P} k}$ depends algebraically on $f$.

COROLlary 6.5. - There are only finitely many analytic subsets of $X$ which are totally invariant by $g$. In particular, there is only a finite number of analytic subsets of $\mathbb{P}^{k}$ which are totally invariant by $f$.

Proof. - We only have to consider totally invariant analytic sets $E$ of pure dimension $q$. The proof is by induction on the dimension $p$ of $X$. Assume that the corollary is true for $X$ of dimension $\leq p-1$ and consider the case of dimension $p$. If $q=p$ then $E$ is a union of components of $X$. There is only a finite number of such analytic sets. If $q<p$, by Theorem $6.1, E$ is contained in $\mathscr{E}_{X}$. Applying the hypothesis of induction to the restriction of $f$ to $\mathscr{E}_{X}$ gives the result.

We now give another characterization of $\mathcal{E}_{X}$. Recall that $\mu_{X}:=T^{p} \wedge[X]$. This is a positive measure of mass $s:=\operatorname{deg} X$. The invariance of $T$ implies that $\mu$ is totally invariant by $g^{m}$, that is, $\left(g^{m}\right)^{*}(\mu)=d^{p m} \mu$. Since $g^{m}$ fixes the components of $X$, we can apply to each component the following result where the second assertion was proved by the authors in [9].

Theorem 6.6. - Assume that $X$ is irreducible. Let $\delta_{a}$ denote the Dirac mass at a point $a \in X$. Then $d^{-p m n}\left(g^{m n}\right)^{*}\left(\delta_{a}\right)$ converge to $s^{-1} \mu_{X}$ if and only if a is out of $\mathscr{E}_{X}$. In particular, if a is a point in $\mathbb{P}^{k}$ then $d^{-k n}\left(f^{n}\right)^{*}\left(\delta_{a}\right)$ converge to $\mu$ if and only if a is out of $\mathscr{E}_{\mathbb{P}}$.

Since $T$ has continuous local potentials, $\mu_{X}$ has no mass on proper analytic subsets of $X$. It follows that if $a \in \mathscr{E}_{X}$, any limit value of $d^{-p m n}\left(g^{m n}\right)^{*}\left(\delta_{a}\right)$ has support in $\mathscr{E}_{X}$ and is singular with respect to $\mu_{X}$. Consider a point $a$ in $X \backslash \mathscr{E}_{X}$. We only have to check the convergence to $s^{-1} \mu_{X}$. Fornæss and the second author proved this convergence for $X=\mathbb{P}^{k}$ 
and for $a$ outside a pluripolar set [16]. Briend and Duval extended this result to $a$ outside the orbit of the critical set of $f$ [1]. They also proposed a geometrical approach in order to prove this property for $a$ outside an analytic set but there is a problem with the counting of multiplicity in their lemma in [1, p.149].

Briend-Duval result can be extended to our situation: for $a$ outside the orbit of $Y$ we have $d^{-p m n}\left(g^{m n}\right)^{*}\left(\delta_{a}\right) \rightarrow s^{-1} \mu_{X}$. We recall the following proposition, see [1] and also [9, 7, 8] for more general cases, in particular, for non-projective manifolds.

Proposition 6.7. - For any $\varepsilon>0$, there is an integer $n_{\varepsilon} \geq 0$ such that if $a$ is out of the analytic set $Y_{\varepsilon}:=Y \cup g^{m}(Y) \cup \cdots \cup g^{m n_{\varepsilon}}(Y)$, then any limit value $\nu$ of $d^{-p m n}\left(g^{m n}\right)^{*}\left(\delta_{a}\right)$ satisfies $\left\|\nu-s^{-1} \mu_{X}\right\| \leq \varepsilon$, where $\|\cdot\|$ denotes the measure mass.

Observe that if $n \geq r \geq 0$ then

$$
d^{-p m n}\left(g^{m n}\right)^{*}\left(\delta_{a}\right)=d^{-p m r} \sum_{b \in g^{-m r}(a)} d^{-p m(n-r)}\left(g^{m(n-r)}\right)^{*}\left(\delta_{b}\right),
$$

where the points in $g^{-m r}(a)$ are counted with multiplicities. So, if a point $a$ does not satisfy the conclusion of Proposition 6.7 then it admits many preimages in $Y_{\varepsilon}$. We quantify now this property.

Let $N_{n}(a)$ denote the number of orbits of $g^{m}$

$$
\mathscr{O}=\left\{a_{-n}, \ldots, a_{-1}, a_{0}\right\}
$$

with $g^{m}\left(a_{-i-1}\right)=a_{-i}$ and $a_{0}=a$ such that $a_{-i} \in Y_{\varepsilon}$ for every $i$. Here, the orbits are counted with multiplicities. So, $N_{n}(a)$ is the number of negative orbits of order $n$ of $a$ which stay in $Y_{\varepsilon}$. Observe that the sequence of functions $\tau_{n}:=d^{-p m n} N_{n}$ decreases to some function $\tau$. Since $\tau_{n}$ are upper semi-continuous with respect to the Zariski topology and $0 \leq \tau_{n} \leq 1$, the function $\tau$ satisfies the same properties. Observe that $\tau(a)$ is the probability that an infinite negative orbit of $a$ stays in $Y_{\varepsilon}$. The following proposition gives also a characterization of $\mathscr{E}_{X}$.

Proposition 6.8. - The function $\tau$ is the characteristic function of $\mathscr{E}_{X}$, that is, $\tau=1$ on $\mathscr{E}_{X}$ and $\tau=0$ on $X \backslash \mathscr{E}_{X}$

Proof. - Since $\mathscr{E}_{X} \subset Y_{\varepsilon}$ and $\mathscr{E}_{X}$ is totally invariant by $g$, we have $\mathscr{E}_{X} \subset\{\tau=1\}$. Let $\theta \geq 0$ denote the maximal value of $\tau$ on $X \backslash \mathscr{E}_{X}$. This value exists since $\tau$ is upper semicontinuous with respect to the Zariski topology (indeed, it is enough to consider the algebraic subset $\left\{\tau \geq \theta_{0}\right\}$ of $X$ which decreases when $\theta_{0}$ increases). We have to check that $\theta=0$. Assume in order to obtain a contradiction that $\theta>0$. Since $\tau \leq 1$, we always have $\theta \leq 1$. Consider the non-empty analytic set $E:=\tau^{-1}(\theta) \backslash \mathscr{E}_{X}$ in $Y_{\varepsilon}$. Let $a^{\prime}$ be a point in $E$. Since $\mathscr{E}_{X}$ is totally invariant, we have $g^{-m}\left(a^{\prime}\right) \cap \mathscr{E}_{X}=\varnothing$. Hence, $\tau\left(b^{\prime}\right) \leq \theta$ for every $b^{\prime} \in g^{-m}(a)$. We deduce from the definition of $\tau$ and $\theta$ that

$$
\theta=\tau\left(a^{\prime}\right) \leq d^{-p m} \sum_{b^{\prime} \in g^{-m}\left(a^{\prime}\right)} \tau\left(b^{\prime}\right) \leq \theta .
$$

It follows that $g^{-m}\left(a^{\prime}\right) \subset E$. Therefore, the analytic subset $\bar{E}$ of $Y_{\varepsilon}$ satisfies $g^{-m}(\bar{E}) \subset \bar{E}$. This contradicts the maximality of $\mathscr{E}_{X}$. 
End of the proof of Theorem 6.6. Let $a$ be a point outside $\mathscr{E}_{X}$. Fix $\varepsilon>0$ and a constant $\alpha>0$ small enough. If $\nu$ is a limit value of $d^{-p m n}\left(g^{m n}\right)^{*}\left(\delta_{a}\right)$, it is enough to show that $\left\|\nu-s^{-1} \mu_{X}\right\| \leq 2 \alpha+\varepsilon$. Proposition 6.8 implies that $\tau(a)=0$. So for $r$ large enough we have $\tau_{r}(a) \leq \alpha$. Consider all the negative orbits $\mathscr{O}_{j}$ of order $r_{j} \leq r$

$$
\mathscr{O}_{j}=\left\{a_{-r_{j}}^{(j)}, \ldots, a_{-1}^{(j)}, a_{0}^{(j)}\right\}
$$

with $g^{m}\left(a_{-i-1}^{(j)}\right)=a_{-i}^{(j)}$ and $a_{0}^{(j)}=a$ such that $a_{-r_{j}}^{(j)} \notin Y_{\varepsilon}$ and $a_{-i}^{(j)} \in Y_{\varepsilon}$ for $i \neq r_{j}$. Each orbit is repeated according to its multiplicity. Let $S_{r}$ denote the family of points $b \in g^{-m r}(a)$ such that $g^{m i}(b) \in Y_{\varepsilon}$ for $0 \leq i \leq r$. Then $g^{-m r}(a) \backslash S_{r}$ consists of the preimages of the points $a_{-r_{j}}^{(j)}$. So, by definition of $\tau_{r}$, we have

$$
d^{-p m r} \# S_{r}=\tau_{r}(a) \leq \alpha
$$

and

$$
d^{-p m r} \#\left(g^{-m r}(a) \backslash S_{r}\right)=d^{-p m r} \sum_{j} d^{p m\left(r-r_{j}\right)}=1-\tau_{r}(a) \geq 1-\alpha .
$$

We have for $n \geq r$

$$
d^{-p m n}\left(g^{m n}\right)^{*}\left(\delta_{a}\right)=d^{-p m n} \sum_{b \in S_{r}}\left(g^{m(n-r)}\right)^{*}\left(\delta_{b}\right)+d^{-p m n} \sum_{j}\left(g^{m\left(n-r_{j}\right)}\right)^{*}\left(\delta_{a_{-r_{j}}^{(j)}}\right) .
$$

Since $d^{-p m n}\left(g^{m n}\right)^{*}$ preserves the mass of any measure, the first term in the last sum is of mass $d^{-p m r} \# S_{r}=\tau_{r}(a) \leq \alpha$ and the second term is of mass $\geq 1-\alpha$. We apply Proposition 6.7 to the Dirac masses at $a_{-r_{j}}^{(j)}$. We deduce that if $\nu$ is a limit value of $d^{-p m n}\left(g^{m n}\right)^{*}\left(\delta_{a}\right)$ then

$$
\left\|\nu-s^{-1} \mu_{X}\right\| \leq 2 \alpha+(1-\alpha) \varepsilon \leq 2 \alpha+\varepsilon .
$$

This completes the proof of the theorem.

COROLlary 6.9. - The cone of positive measures on $X$ which are totally invariant by $g^{m}$, is of finite dimension. In particular, the cone of positive measures on $\mathbb{P}^{k}$ which are totally invariant by $f$, is of finite dimension.

Proof. - Replacing $f$ by an iterate allows to assume that $g^{m}$ fixes all the components of every analytic set which is totally invariant by $g^{m}$. So, all these components are totally invariant. Let $\nu$ be an extremal probability measure totally invariant by $g^{m}$. Let $X^{\prime}$ be the smallest analytic set totally invariant by $g^{m}$ such that $\nu\left(X^{\prime}\right)=1$. Since $\nu$ is extremal, $X^{\prime}$ is irreducible and $\nu\left(\mathscr{E}_{X^{\prime}}\right)=0$. It follows from Theorem 6.6 and the invariance of $\nu$ that $\nu$ is proportional to $\mu_{X^{\prime}}$. By Corollary 6.5 , the family of such measures is finite.

The following lemma will be useful in the proof of our main results where $n_{0}$ is an index such that $\mathscr{E}_{X}^{n}=\mathscr{E}_{X}$ for $n \geq n_{0}$.

Lemma 6.10. - There is a constant $\theta>0$ such that if $Z$ is an analytic subset of pure dimension $q \leq p-1$ of $X$ not contained in $\mathscr{E}_{X}$ then for every $n \geq 0, g^{-m n}(Z)$ contains an analytic set $Z_{-n}$ of pure dimension $q$ of degree $\geq \theta d^{m n(p-q)}$. Moreover, if $n \geq n_{0}$ and if $x$ is a generic point in $Z_{-n}$, then $x \in \operatorname{reg}(X), g^{m\left(n-n_{0}\right)}(x) \in \operatorname{reg}(X)$ and $g^{m\left(n-n_{0}\right)}$ defines a biholomorphism between a neighbourhood of $x$ and a neighbourhood of $g^{m\left(n-n_{0}\right)}(x)$ in $X$. 
Proof. - Let $P$ be a generic projective plane in $\mathbb{P}^{k}$ of dimension $k-q$. Consider a point $a$ in $Z \cap P \backslash \mathscr{E}_{X}$. Since $\mathscr{E}_{X}=\mathscr{E}_{X}^{n_{0}}$, we have $g^{-m l}(a) \not \subset E_{1}$ for some $0 \leq l \leq n_{0}$. Then,

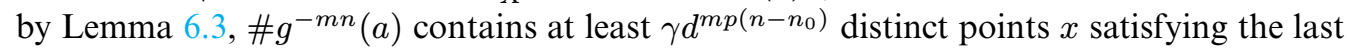
property in the lemma. Let $Z_{-n}$ denote the union of the irreducible components of $g^{-m n}(Z)$ which contain at least one such point $x$. Then, $Z_{-n}$ satisfies the last property in the lemma. We have $\# Z_{-n} \cap f^{-m n}(P) \geq \gamma d^{m p\left(n-n_{0}\right)}$. Since $\operatorname{deg} f^{-m n}(P)=d^{m n q}$, we obtain that $\operatorname{deg} Z_{-n} \geq \theta d^{m(p-q) n}$ for $\theta:=\gamma d^{-m p n_{0}}$.

\section{Convergence towards the Green current}

In this section, we will prove the main results. Define the exceptional set $\mathscr{E}$ as the union of proper analytic subsets $E$ of $\mathbb{P}^{k}$ which are totally invariant by $f$ and are minimal in the following sense. The set $E$ does not contain non-empty proper analytic sets which are totally invariant by $f$. Theorem 6.1 and Corollary 6.5 imply that $\mathscr{E}$ is a totally invariant analytic set and it does not change if we replace $f$ by an iterate of $f$, see also Remark 6.4. We have the following result which implies Theorems 1.1 and 1.2.

TheOREM 7.1. - Let $f, T, \mathscr{E}$ be as above. Let $\mathscr{G}$ be a family of modulo $T$ psh functions on $\mathbb{P}^{k}$ which is bounded in $L^{1}\left(\mathbb{P}^{k}\right)$. Assume that the restriction of $\mathscr{G}$ to each component of $\mathscr{E}$ is a bounded family of modulo $T$ wpsh functions. Then, $d^{-n} u \circ f^{n}$ converge to 0 in $L^{1}\left(\mathbb{P}^{k}\right)$ uniformly on $u \in \mathscr{G}$.

Let $m \geq 1$ be an integer such that $f^{m}$ fixes all the irreducible components of all the totally invariant analytic sets. By Proposition 5.7, we can replace $f$ by $f^{m}$ and assume that $f$ fixes all these components. Let $X_{p}$ denote the union of totally invariant sets of pure dimension $p$. We will prove by induction on $p$ that $d^{-n} u \circ f^{n}$ converge to 0 in $L^{1}\left(X_{p}\right)$ uniformly on $u \in \mathscr{G}$. We obtain the theorem for $p=k$ and $X_{k}=\mathbb{P}^{k}$. Assume this convergence on $X_{0}, \ldots, X_{p-1}$ (the case $p=0$ is clear). Define $X:=X_{p}$ and $\mathscr{E}_{X}$ as in Section 6 . Note that if $X$ is reducible at a point $x \in X$ then $x$ belongs to $\mathscr{E}_{X}$. From the induction hypothesis, on each component $E$ of $\mathscr{E}_{X}, d^{-n} u \circ f^{n}$ converge in $L^{1}$ to 0 uniformly on $u \in \mathscr{G}$. We deduce that $\mathscr{G}$ is bounded in $L^{1}(E)$. So, if $Z$ is a component of $X$, which is not minimal in the above sense, by Lemma 3.5, $\mathscr{G}$ is bounded in $L^{1}(Z)$. If $Z$ is a minimal component of $X$, then by hypothesis of the theorem, $\mathscr{G}$ is bounded in $L^{1}(Z)$. So, we can apply Corollary 3.9 to $\mathscr{G}$.

Let $\mathscr{G}^{\prime}$ denote the set of all the modulo $T$ wpsh functions on $X$ which are limit values in $L^{1}(X)$ of a sequence $\left(d^{-n} u_{n} \circ f^{n}\right)$ with $u_{n} \in \mathscr{G}$. For every $u \in \mathscr{G}^{\prime}$, Corollary 3.9 implies that $u \leq 0$. Since $\mathscr{E}_{X} \subset X$, by induction hypothesis we have convergence on $\mathscr{E}_{X}$. The last assertion of Proposition 3.3 implies that $u \geq 0$ on $\mathscr{E}_{X}$. Hence, $u=0$ on $\mathscr{E}_{X}$ for every $u \in \mathscr{G}^{\prime}$. We claim that $u$ is (strongly) psh modulo $T$ at every point of $\mathscr{E}_{X}$, that is, if $\tau:(\mathbb{C}, 0) \rightarrow X$ is a germ of holomorphic disc with $\tau(0) \in \mathscr{E}_{X}$ then $u \circ \tau$ is subharmonic modulo $\tau^{*}(T)$. Let $\pi: \widetilde{X} \rightarrow X \subset \mathbb{P}^{k}$ be a desingularization of $X$ and $\widetilde{u}$ the lift of $u$ to $\widetilde{X}$ which is psh modulo $\pi^{*}(T)$. Since $d^{-n} u_{n} \circ f^{n}$ are psh modulo $T, \widetilde{u}$ vanishes on irreductible analytic sets $\widetilde{E}$ such that $\pi(\widetilde{E})$ is contained in $\mathscr{E}_{X}$ and is totally invariant. So, if $E \subset \mathscr{E}_{X}$ is analytic and totally invariant, then $\widetilde{u}$ vanishes on $\pi^{-1}\left(E^{\prime}\right)$ for some dense Zariski open subset of $E$. Therefore, $u$ is psh modulo $T$ at every point of $E^{\prime}$. This and Lemma 7.2 below applied to $v_{n}$ and to 
$v_{0}:=u$ imply the claim. It is clear that $\mathscr{G}^{\prime}$ is compact. Fix a function $v_{0} \in \mathscr{G}^{\prime}$. We have to show that $v_{0}=0$.

Lemma 7.2. - There are functions $v_{n} \in \mathscr{G}^{\prime}$ such that $v_{n+1}=d^{-1} v_{n} \circ f$ almost everywhere for $n \in \mathbb{Z}$.

Proof. - Assume that $v_{0}$ is the limit of a sequence $\left(d^{-n_{i}} u_{n_{i}} \circ f^{n_{i}}\right)$. Then, for $n \geq 0$ the sequence $\left(d^{-n_{i}-n} u_{n_{i}} \circ f^{n_{i}+n}\right)$ converges to $d^{-n} v_{0} \circ f^{n}$. Lemma 3.8 implies that $d^{-n} v_{0} \circ f^{n}$ is equal almost everywhere to an element $v_{n}$ of $\mathscr{G}^{\prime}$. If $v_{-1} \in \mathscr{G}^{\prime}$ is a limit value of $\left(d^{-n_{i}+1} u_{n_{i}}\right.$ 。 $f^{n_{i}-1}$ ) then $v_{0}=d^{-1} v_{-1} \circ f$ almost everywhere. We construct the functions $v_{-n}$ in the same way by induction. If $v_{-n}$ is the limit of $\left(d^{-m_{i}} u_{m_{i}}^{\prime} \circ f^{m_{i}}\right)$ then we obtain $v_{-n-1}$ as a limit value of $\left(d^{-m_{i}+1} u_{m_{i}}^{\prime} \circ f^{m_{i}-1}\right)$.

Proof of Theorem 7.1. Let $\mathscr{G}^{\prime \prime}$ denote the set of all the modulo $T$ wpsh functions $w$ on $X$ which are limit values of the sequence $\left(v_{-n}\right)_{n \geq 0}$. Since $\mathscr{G}^{\prime}$ is compact, we have $\mathscr{G}^{\prime \prime} \subset \mathscr{G}^{\prime}$. We have to show that $v_{0}=0$. Assume this is not the case. Since $v_{0}=d^{-n} v_{-n} \circ f^{n}$ almost everywhere, by Lemma 5.10, there is a constant $\alpha_{0}>0$ such that $\max _{X} \nu_{X}(w, a) \geq \alpha_{0}$ for every $w \in \mathscr{G}^{\prime \prime}$. Fix a function $w_{0} \in \mathscr{G}^{\prime \prime}$.

Lemma 7.3. - There are functions $w_{n} \in \mathscr{G}^{\prime \prime}$ such that $w_{n+1}=d^{-1} w_{n} \circ f$ almost everywhere for $n \in \mathbb{Z}$.

Proof. - Assume that $w_{0}$ is the limit of $\left(v_{-n_{i}}\right)$. Let $w_{1}$ and $w_{-1}$ be modulo $T$ wpsh functions which are limit values of $\left(v_{-n_{i}+1}\right)$ and $\left(v_{-n_{i}-1}\right)$ respectively. These functions belong to $\mathscr{G}^{\prime \prime}$. Then, $w_{0}=d^{-1} w_{-1} \circ f$ and $w_{1}=d^{-1} w_{0} \circ f$ almost everywhere. We obtain the lemma by induction. If $w_{n}$ is the limit value of $\left(v_{-m_{i}}\right)$ then we obtain $w_{n-1}$ or $w_{n+1}$ as limit values of $\left(v_{-m_{i}-1}\right)$ or $\left(v_{-m_{i}+1}\right)$ respectively.

For $\alpha>0$ and $0 \leq q \leq p-1$, denote by $\mathbb{N}_{\alpha, q}$ (resp. $\mathbb{N}_{\alpha,>q}$ ) the family of indices $n \in \mathbb{N}$ such that $\left\{\nu_{X}\left(w_{-n}, a\right) \geq \alpha\right\}$ is a non-empty analytic set of dimension $q$ (resp. $>q$ ). From the definition of $\mathscr{G}^{\prime \prime}$, we have $\cup_{q} \mathbb{N}_{\alpha_{0}, q}=\mathbb{N}$. Hence, there is a maximal integer $q$ such that the upper density

$$
\Theta^{*}\left(\mathbb{N}_{\alpha, q}\right):=\limsup _{n \rightarrow \infty} \frac{\# \mathbb{N}_{\alpha, q} \cap\{0, \ldots, n-1\}}{n}
$$

is strictly positive for some constant $\alpha>0$. Fix a constant $0<\beta \ll \alpha$ that we will choose later. The maximality of $q$ implies that $\Theta^{*}\left(\mathbb{N}_{\beta,>q}\right)=0$. It follows that

$$
\delta:=\Theta^{*}\left(\mathbb{N}_{\alpha, q} \backslash \mathbb{N}_{\beta,>q}\right)=\Theta^{*}\left(\mathbb{N}_{\alpha, q}\right)>0 .
$$

Hence, for any integer $l \geq 1$, there is an integer $n_{1} \in \mathbb{N}_{\alpha, q} \backslash \mathbb{N}_{\beta,>q}$ such that

$$
\#\left(\mathbb{N}_{\alpha, q} \backslash \mathbb{N}_{\beta,>q}\right) \cap\left\{n_{1}, \ldots, n_{1}+l\right\} \geq \frac{1}{2} \delta l .
$$

Fix $l$ large enough and choose $\beta=\frac{1}{2} d^{-l-l k^{2}} \alpha$. Replacing $w_{0}$ by $w_{-n_{1}}$ allows us to assume that $n_{1}=0$. This simplifies the notation. We are looking for a contradiction using Lemma 4.6 applied to $u:=w_{0}$. The hypothesis on the dimension of $\left\{\nu\left(w_{0}, a\right)>\beta\right\}$ is satisfied since $0 \in \mathbb{N}_{\alpha, q} \backslash \mathbb{N}_{\beta,>q}$. Let $n_{0}$ be given in Theorem 6.1 and Lemma 6.10. Choose integers $n_{0}<i_{1}<\cdots<i_{s} \leq l$, with $s \geq \frac{1}{2} \delta l-n_{0}-1$, in $\mathbb{N}_{\alpha, q} \backslash \mathbb{N}_{\beta,>q}$. Let $Z_{r}^{\prime \prime}$ be an irreducible analytic set of dimension $q$ such that $\nu_{X}\left(w_{-i_{r}}, x\right) \geq \alpha$ on $Z_{r}^{\prime \prime}$. We have seen that $w_{-i_{r}}$ is 
(strongly) psh modulo $T$ at every point in $\mathscr{E}_{X}$ and vanishes there. Hence, its Lelong number vanishes on $\mathscr{E}_{X}$ and $Z_{r}^{\prime \prime} \not \subset \mathscr{E}_{X}$. By Lemma 6.10 (we assumed that $m=1$ ), there are analytic sets $Z_{r} \subset g^{-i_{r}}\left(Z_{r}^{\prime \prime}\right)$ of pure dimension $q$ and of degree $\geq \theta d^{i_{r}(p-q)}=: d_{r}$ such that if $x$ is a generic point in $Z_{r}$ then $x \in \operatorname{reg}(X), x^{\prime}:=g^{i_{r}-n_{0}}(x) \in \operatorname{reg}(X)$ and $g^{i_{r}-n_{0}}$ defines a biholomorphism between neighbourhoods of $x$ and $x^{\prime}$. We now check the assumption of Lemma 4.6 that the Lelong number of $w_{0}$ is $\geq 2 \beta$ on $Z_{r}$.

Since $w_{0}=d^{-i_{r}+n_{0}} w_{-i_{r}+n_{0}} \circ g^{i_{r}-n_{0}}$, we deduce from the previous property of $g^{i_{r}-n_{0}}$ that

$$
\nu_{X}\left(w_{0}, x\right)=d^{-i_{r}+n_{0}} \nu_{X}\left(w_{-i_{r}+n_{0}}, x^{\prime}\right) .
$$

Define $x^{\prime \prime}:=g^{i_{r}}(x)=g^{n_{0}}\left(x^{\prime}\right)$. This is a point in $Z_{r}^{\prime \prime}$. The local topological degree of $f^{n_{0}}$ at $x^{\prime}$ is $\leq d^{n_{0} k}$. Proposition 4.1 applied to $h:=f^{n_{0}}$ and the identity $w_{-i_{r}+n_{0}}=d^{-n_{0}} w_{-i_{r}} \circ g^{n_{0}}$ imply that

$$
\nu_{X}\left(w_{-i_{r}+n_{0}}, x^{\prime}\right) \geq d^{-n_{0}-n_{0} k^{2}} \nu_{X}\left(w_{-i_{r}}, x^{\prime \prime}\right) \geq d^{-n_{0}-n_{0} k^{2}} \alpha .
$$

It follows that $\nu_{X}\left(w_{0}, x\right) \geq d^{-i_{r}-n_{0} k^{2}} \alpha=: \nu_{r} \geq 2 \beta$. Applying Lemma 4.6 yields

$$
\theta d^{-n_{0} k^{2}(p-q)} \alpha^{p-q} s \leq 2^{p-q+1} \operatorname{deg}(X)^{p-q} .
$$

This is a contradiction if $l$ is large enough, since $s \geq \frac{1}{2} \delta l-n_{0}-1$.

Proof of Theorem 1.3. It is enough to prove that for $f$ generic in $\mathcal{H}_{d}$ we have $\mathscr{E}_{\mathbb{P} k}=\varnothing$. By Lemma 6.2 applied to $X=\mathscr{E}_{\mathbb{P}^{k}}$, it is enough to show that if $f$ is generic, $\lim \sup d^{-(k-1) n} \#$ $f^{-n}(x)=+\infty$ for every $x \in \mathbb{P}^{k}$. Here, we count points without multiplicity. Fix an $m \geq 1$ such that $d^{m}>2^{k} k$ !. We show for $f$ generic that $\# f^{-m}(x)>d^{m(k-1)}$ for every $x \in \mathbb{P}^{k}$. This implies the result. Observe that the family of such $f$ is a Zariski open set in $\mathcal{H}_{d}$. So, it is enough to construct an $f$ satisfying this property.

Choose a rational map $h: \mathbb{P}^{1} \rightarrow \mathbb{P}^{1}$ of degree $d$ such that $\# h^{-m}(x) \geq \frac{1}{2} d^{m}$ for every $x \in \mathbb{P}^{1}$. To this end, it is enough to take a map $h$ whose critical points are simple and have disjoint orbits. Now, construct the map $f$ using an idea of Ueda [32]. Let $\pi: \mathbb{P}^{1} \times \cdots \times \mathbb{P}^{1} \rightarrow$ $\mathbb{P}^{k}$ denote the canonical map which identifies all points $\left(x_{1}, \ldots, x_{k}\right)$ with the points obtained by permutation of coordinates. If $\widehat{f}$ is the endomorphism of $\mathbb{P}^{1} \times \cdots \times \mathbb{P}^{1}, k$ times, defined by $\widehat{f}\left(x_{1}, \ldots, x_{k}\right):=\left(h\left(x_{1}\right), \ldots, h\left(x_{k}\right)\right)$, then there is a holomorphic map $f: \mathbb{P}^{k} \rightarrow \mathbb{P}^{k}$ of algebraic degree $d$ such that $f \circ \pi=\pi \circ \widehat{f}$. We also have $f^{m} \circ \pi=\pi \circ \widehat{f}^{m}$. Consider a point $x$ in $\mathbb{P}^{k}$ and a point $\widehat{x}$ in $\pi^{-1}(x)$. We have $\pi^{-1}\left(f^{-m}(x)\right)=\widehat{f}^{-m}\left(\pi^{-1}(x)\right)$. Hence, $\# \pi^{-1}\left(f^{-m}(x)\right) \geq \# \widehat{f}^{-m}(\widehat{x}) \geq 2^{-k} d^{m k}$. Since $\pi$ has degree $k$ !, we obtain $\# f^{-m}(x) \geq$ $\frac{1}{2^{k} k !} d^{m k}>d^{m(k-1)}$. This completes the proof.

ReMARK 7.4. - Let $\mathscr{C}$ denote the compact convex set of totally invariant $(1,1)$-currents of mass 1 on $\mathbb{P}^{k}$. Define an operator $\vee$ on $\mathscr{C}$. If $S_{1}, S_{2}$ are elements of $\mathscr{C}$, write $S_{i}=T+d d^{c} u_{i}$ with $u_{i}$ psh modulo $T$ on $\mathbb{P}^{k}$ such that $u_{i} \leq 0$ and $u_{i}=0$ on $\operatorname{supp}(\mu)$, see Corollary 3.9. Define $S_{1} \vee S_{2}:=T+d d^{c} \max \left(u_{1}, u_{2}\right)$. It is easy to check that $S_{1} \vee S_{2}$ is an element of $\mathscr{C}$. An element $S$ is said to be minimal if $S=S_{1} \vee S_{2}$ implies $S_{1}=S_{2}=S$. It is clear that $T$ is not minimal if $\mathscr{C}$ contains other currents. A current of integration on a totally invariant hypersurface is a minimal element. 
EXAMPLE 7.5. - Let $\left[z_{0}: \cdots: z_{k}\right]$ denote the homogeneous coordinates of $\mathbb{P}^{k}$ and $\pi:$ $\mathbb{C}^{k+1} \backslash\{0\} \rightarrow \mathbb{P}^{k}$ the canonical projection. Consider the map $f\left[z_{0}: \cdots: z_{k}\right]:=\left[z_{0}^{d}: \cdots: z_{k}^{d}\right]$, $d \geq 2$. The Green $(1,1)$-current $T$ of $f$ is given by $\pi^{*}(T)=d d^{c}\left(\max _{i} \log \left|z_{i}\right|\right)$, see [29], or equivalently $T=\omega+d d^{c} v$ where

$$
v\left[z_{0}: \cdots: z_{k}\right]:=\max _{0 \leq i \leq k} \log \left|z_{i}\right|-\frac{1}{2} \log \left(\left|z_{0}\right|^{2}+\cdots+\left|z_{k}\right|^{2}\right) .
$$

The currents $T_{i}$ of integration on $\left(z_{i}=0\right)$ belong to $\mathscr{C}$ and $T_{j}=T+d d^{c} u_{j}$ with $u_{j}:=$ $\log \left|z_{j}\right|-\max _{i} \log \left|z_{i}\right|$. These currents are minimal. If $\alpha_{0}, \ldots, \alpha_{k}$ are positive real numbers such that $\alpha:=1-\sum \alpha_{i}$ is positive, then $S:=\alpha T+\sum \alpha_{i} T_{i}$ is an element of $\mathscr{C}$. We have $S=T+d d^{c} u$ with $u:=\sum \alpha_{i} u_{i}$. The current $S$ is minimal if and only if $\alpha=0$. One can obtain other elements of $\mathscr{C}$ using the operator $\vee$. One can also prove that $\mathscr{C}$ admits an infinite number of elements which are extremal in the cone of positive closed $(1,1)$-currents. This implies that $\mathscr{C}$ has infinite dimension. For example, in dimension 2, the currents associated to

$$
u\left(\left[z_{0}: z_{1}: z_{2}\right]\right):=\max \left(\log \left|z_{0}\right|\left|z_{1}\right|^{\alpha-1}, \alpha \log \left|z_{2}\right|\right)-\frac{\alpha}{2} \log \left(\left|z_{0}\right|^{2}+\left|z_{1}\right|^{2}+\left|z_{2}\right|^{2}\right), \quad \alpha>1,
$$

are extremal. The elements of the set $\mathscr{E}$ in this case are just the points $[0: \cdots: 0: 1: 0: \cdots: 0]$.

\section{Polynomial automorphisms}

The approach that we used above can be extended to other situations. From now on we consider a polynomial automorphism $f: \mathbb{C}^{k} \rightarrow \mathbb{C}^{k}$ of degree $\geq 2$ and its extension as a birational map on $\mathbb{P}^{k}$ that we also denote by $f$. Let $I_{+}$and $I_{-}$denote the indeterminacy sets of $f$ and $f^{-1}$ respectively. These are the analytic sets where $f$ and $f^{-1}$ are not defined; they are contained in the hyperplane at infinity $L:=\mathbb{P}^{k} \backslash \mathbb{C}^{k}$. Assume that $f$ is regular, i.e. $I_{+} \cap$ $I_{-}=\varnothing$. We refer the reader to [29] for the basic properties of regular automorphisms. There is an integer $1 \leq s \leq k-1$ such that $I_{+}$and $I_{-}$are irreducible analytic sets of dimension $s-1$ and $k-s-1$ respectively. We also have $f\left(L \backslash I_{+}\right)=f\left(I_{-}\right)=I_{-}$and $f^{-1}\left(L \backslash I_{-}\right)=$ $f^{-1}\left(I_{+}\right)=I_{+}$. The maps $f^{n}$ and $f^{-n}$ are also regular. The algebraic degrees $d_{+}$and $d_{-}$of $f$ and $f^{-1}$ satisfy the relation $d_{+}^{k-s}=d_{-}^{s}$.

The Green currents of bidegree $(1,1)$ associated to $f$ and $f^{-1}$ are denoted by $T_{+}$and $T_{-}$. They are limits in the sense of currents of $d_{+}^{-n}\left(f^{n}\right)^{*}(\omega)$ and $d_{-}^{-n}\left(f^{n}\right)_{*}(\omega)$ respectively. The current $T_{+}$has locally continuous potentials outside $I_{+}$, the current $T_{-}$has locally continuous potentials outside $I_{-}$. We also have $f^{*}\left(T_{+}\right)=d_{+} T_{+}$and $f_{*}\left(T_{-}\right)=d_{-} T_{-}$. We will consider the problem of convergence towards $T_{+}$, the case of $T_{-}$is obtained in the same way.

Let $g: X \rightarrow X$ denote the restriction of $f$ to $X:=I_{-}$. The positive measure $\mu_{X}:=$ $T_{+}^{k-s-1} \wedge[X]$ has positive mass. Since $T_{+}$is totally invariant, we have $g^{*}\left(\mu_{X}\right)=d_{+}^{k-s-1} \mu_{X}$. This implies that $g$ has topological degree $d_{+}^{k-s-1}$. We construct as above the families $X_{0}$, $\ldots, X_{k-s-1}$ of totally invariant sets associated to $g$ with $X_{k-s-1}=I_{-}$. Let $\mathscr{E}_{+}$denote the union of minimal components in $\left\{X_{0}, \ldots, X_{k-s-1}\right\}$. We have the following result, see [16] for the case of dimension 2 . 
Theorem 8.1. - Let $S$ be a positive closed $(1,1)$-current of mass 1 on $\mathbb{P}^{k}$. Assume that the local potentials of $S$ are not identically equal to $-\infty$ on any irreducible component of $\mathscr{E}_{+}$. Then, $d_{+}^{-n}\left(f^{n}\right)^{*}(S)$ converge to $T_{+}$.

The proof follows the same lines as above. We will describe the difference with the case of holomorphic endomorphisms and leave the details to the reader. There is a neighbourhood $V$ of $I_{+}$with smooth boundary, which can be chosen arbitrarily small, such that $f\left(\mathbb{P}^{k} \backslash V\right) \Subset$ $\mathbb{P}^{k} \backslash V$, see [29]. If $S$ is as above, there is a modulo $T_{+}$psh function $u$ such that $S=T_{+}+d d^{c} u$. This function is defined and is locally bounded from above on $\mathbb{P}^{k} \backslash I_{+}$. Denote by $\mathscr{G}$ the set of modulo $T_{+}$psh functions on $\mathbb{P}^{k}$ which are limit values of $d_{+}^{-n} u \circ f^{n}$. Since the Lelong number of $u$ is $\leq 1$ at every point in $\mathbb{P}^{k} \backslash I_{+}$and since $f$ is an automorphism, Proposition 4.1 implies that the Lelong number of $d_{+}^{-n} u \circ f^{n}$ is $\leq d_{+}^{-n}$ at every point in $\mathbb{C}^{k}$.

On the other hand, for $v \in \mathscr{G}$, we prove as in the previous sections that $v \leq 0$ and $v=0$ on $X=I_{-}$. It follows that $v=0$ on $L \backslash I_{+}$since we can write $v=d_{+}^{-1} v^{\prime} \circ f$ with $v^{\prime} \in \mathscr{G}$ and $f\left(L \backslash I_{+}\right)=I_{-}$. The upper semi-continuity of the Lelong number implies that for every $\delta>0$, there is an $m$ such that the Lelong number of $d_{+}^{-m} u \circ f^{m}$ is smaller than $\delta$ on $\mathbb{P}^{k} \backslash V$. We want to prove that $v=0$ on $\mathbb{P}^{k} \backslash \bar{V}$.

Assume that $v=\lim d_{+}^{-n_{i}} u \circ f^{n_{i}}$ and that $v \leq-2 \alpha$ with $\alpha>0$, on a ball $B \subset \mathbb{P}^{k} \backslash \bar{V}$ of radius $r$. Then as in Proposition 5.7, we will have that $d_{+}^{-m} u \circ f^{m} \leq-d_{+}^{n_{i}-m} \alpha$ on a ball $B_{i} \subset \mathbb{P}^{k} \backslash \bar{V}$ of radius $\exp \left(-c r^{-2 k} d_{+}^{n_{i}-m}\right)$; this contradicts Proposition 4.2 for $\delta$ small and $n_{i}$ large. We can also obtain a uniform convergence for regular automorphisms as in Theorem 7.1.

\section{REFERENCES}

[1] J.-Y. BRIEND, J. Duval, Deux caractérisations de la mesure d'équilibre d'un endomorphisme de $\mathrm{P}^{k}(\mathbf{C})$, Publ. Math. Inst. Hautes Études Sci. 93 (2001), 145-159.

[2] H. Brolin, Invariant sets under iteration of rational functions, Ark. Mat. 6 (1965), 103-144.

[3] D. Cerveau, A. Lins Neto, Hypersurfaces exceptionnelles des endomorphismes de CP (n), Bol. Soc. Brasil. Mat. (N.S.) 31 (2000), 155-161.

[4] S. S. Chern, H. I. Levine, L. Nirenberg, Intrinsic norms on a complex manifold, in Global Analysis (Papers in Honor of K. Kodaira), Univ. Tokyo Press, 1969, 119-139.

[5] J.-P. Demailly, Monge-Ampère operators, Lelong numbers and intersection theory, in Complex analysis and geometry, Univ. Ser. Math., Plenum, 1993, 115-193.

[6] J.-P. Demailly, Complex analytic geometry, http://www-fourier.ujf-grenoble. fr/ demailly/manuscripts/agbook.pdf, 2007.

[7] T.-C. Dinh, Distribution des préimages et des points périodiques d'une correspondance polynomiale, Bull. Soc. Math. France 133 (2005), 363-394.

[8] T.-C. Dinh, Suites d'applications méromorphes multivaluées et courants laminaires, $J$. Geom. Anal. 15 (2005), 207-227.

[9] T.-C. Dinh, N. Sibony, Dynamique des applications d'allure polynomiale, J. Math. Pures Appl. 82 (2003), 367-423. 
[10] T.-C. Dinh, N. Sibony, Distribution des valeurs de transformations méromorphes et applications, Comment. Math. Helv. 81 (2006), 221-258.

[11] T.-C. Dinh, N. Sibony, Pull-back of currents by holomorphic maps, Manuscripta Math. 123 (2007), 357-371.

[12] T.-C. Dinh, N. Sibony, Super-potentials of positive closed currents, intersection theory and dynamics, preprint arXiv:math/0703702, 2007, to appear in Acta Math.

[13] C. Favre, M. Jonsson, Brolin's theorem for curves in two complex dimensions, Ann. Inst. Fourier (Grenoble) 53 (2003), 1461-1501.

[14] C. Favre, M. Jonsson, Eigenvaluations, Ann. Sci. École Norm. Sup. 40 (2007), 309349.

[15] J. E. Forness, R. NARASimhan, The Levi problem on complex spaces with singularities, Math. Ann. 248 (1980), 47-72.

[16] J. E. Fornæss, N. Sibony, Complex Hénon mappings in $\mathbf{C}^{2}$ and Fatou-Bieberbach domains, Duke Math. J. 65 (1992), 345-380.

[17] J. E. Fornæss, N. Sibony, Complex dynamics in higher dimension. I. Complex analytic methods in dynamical systems (Rio de Janeiro, 1992), Astérisque 222 (1994), 5, 201-231.

[18] J. E. Fornæss, N. Sibony, Complex dynamics in higher dimension. II, in Modern methods in complex analysis (Princeton, NJ, 1992), Ann. of Math. Stud. 137, Princeton Univ. Press, 1995, 135-182.

[19] A. Freire, A. Lopes, R. Mañé, An invariant measure for rational maps, Bol. Soc. Brasil. Mat. 14 (1983), 45-62.

[20] V. Guedu, Equidistribution towards the Green current, Bull. Soc. Math. France 131 (2003), 359-372.

[21] V. Guedu, Decay of volumes under iteration of meromorphic mappings, Ann. Inst. Fourier (Grenoble) 54 (2004), 2369-2386.

[22] L. Hörmander, The analysis of linear partial differential operators. I, Grund. Math. Wiss. 256, Springer, 1983.

[23] P. Lelong, Fonctions plurisousharmoniques et formes différentielles positives, Dunod, 1968.

[24] M. J. LuUbich, Entropy properties of rational endomorphisms of the Riemann sphere, Ergodic Theory Dynam. Systems 3 (1983), 351-385.

[25] M. Meo, Image inverse d'un courant positif fermé par une application analytique surjective, C. R. Acad. Sci. Paris Sér. I Math. 322 (1996), 1141-1144.

[26] M. Meo, Inégalités d'auto-intersection pour les courants positifs fermés définis dans les variétés projectives, Ann. Scuola Norm. Sup. Pisa Cl. Sci. 26 (1998), 161-184.

[27] A. RussakovskiI, B. Shiffman, Value distribution for sequences of rational mappings and complex dynamics, Indiana Univ. Math. J. 46 (1997), 897-932.

[28] B. Shiffman, M. Shishikura, T. Ueda, On totally invariant varieties of holomorphic mappings of $\mathbb{P}^{n}$, preprint, 2000.

[29] N. Sibony, Dynamique des applications rationnelles de $\mathbf{P}^{k}$, in Dynamique et géométrie complexes (Lyon, 1997), Panor. Synthèses 8, Soc. Math. France, 1999, 97-185. 
[30] Y. T. SiU, Analyticity of sets associated to Lelong numbers and the extension of closed positive currents, Invent. Math. 27 (1974), 53-156.

[31] J.-C. Tougeron, Idéaux de fonctions différentiables, 71, Springer, 1972.

[32] T. Ueda, Fatou sets in complex dynamics on projective spaces, J. Math. Soc. Japan 46 (1994), 545-555.

[33] G. Vigny, Lelong-Skoda transform for compact Kähler manifolds and selfintersection inequalities, preprint arXiv:0711.3782v1, 2007.

(Manuscrit reçu le 15 novembre 2007; accepté, après révision, le 19 décembre 2007.)

\author{
Tien-Cuong DinH \\ Université Pierre et Marie Curie-Paris 6 \\ UMR 7586 \\ Institut de Mathématiques de Jussieu \\ 175, rue du Chevaleret \\ 75013 Paris, France \\ E-mail: dinh@math.jussieu.fr \\ URL: http://www.math.jussieu.fr/ dinh \\ Nessim Sibony \\ Université Paris-Sud \\ Mathématique \\ Bâtiment 425 \\ 91405 Orsay, France \\ E-mail: nessim.sibony@math.u-psud.fr
}

